\title{
Critical Contaminant/Critical Pathway Analysis - Surface Water Transport for Nonradioactive Contaminants (U)
}

by

K. F. Chen

Westinghouse Savannah River Company

Savannah River Site

Aiken, South Carolina 29808

DOE Contract No. DE-AC09-89SA18035

This paper was prepared in connection with work done under the above contract number with the U.S.

Department of Energy. By acceptance of this paper, the publisher and/or recipient acknowledges the U. S. Government's right to retain a nonexclusive, royalty-free license in and to any copyright covering this paper, along with the right to reproduce and to authorize others to reproduce all or part of the copyrighted paper. 


\section{DISCLAIMER}

This report was prepared as an account of work sponsored by an agency of the United States Government. Neither the United States Government nor any agency thereof, nor any of their employees, makes any warranty, express or implied, or assumes any legal liability or responsibility for the accuracy, completeness, or usefulness of any information, apparatus, product, or process disclosed, or represents that its use would not infringe privately owned rights. Reference herein to any specific commercial product, process, or service by trade name, trademark, manufacturer, or otherwise does not necessarily constitute or imply its endorsement, recommendation, or favoring by the United States Government or any agency thereof. The views and opinions of authors expressed herein do not necessarily state or reflect those of the United States Government or any agency thereof.

This report has been reproduced directly from the best available copy.

Available to DOE and DOE contractors from the Office of Scientific and Technical Information, P.O. Box 62, Oak Ridge, TN 37831; prices available from (615) 576-8401.

Available to the public from the National Technical Information Service, U.S. Department of Commerce; 5285 Port Royal Road, Springfield, VA 22161. 


\section{DISCLAMMER}

Portions of this document may be illegible in electronic image products. Images are produced from the best available original document. 
Waste Management and

Environmental Technology

WSRC-RP-96-555

CRITICAL CONTAMINANT/CRITICAL PATHWAY ANALYSIS - SURFACE WATER TRANSPORT FOR NONRADIOACTIVE CONTAMINANTS (U)

Kuo-Fu Chen

Technical Reviewer

Dam PrLugaze Date October 28,1996

Approval

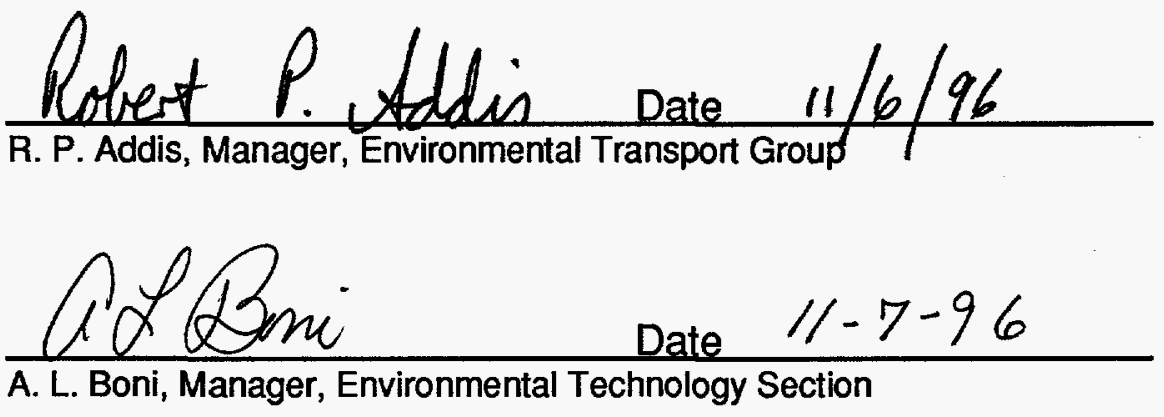

NOVEMBER 1996

Westinghouse Savannah River Company Savannah River Site Aiken, SC 29808

PREPARED FOR THE U.S. DEPARTMENT OF ENERGY UNDER CONTRACT NO. DE-AC09-89SR18035 
Waste Management and

Environmental Technology

CRITICAL CONTAMINANT

OUTFALLS

HEALTH RISK

CANCER

Retention: Lifetime

\title{
CRITICAL CONTAMINANT/CRITICAL PATHWAY ANALYSIS - SURFACE WATER TRANSPORT FOR NONRADIOACTIVE CONTAMINANTS (U)
}

\author{
Kuo-Fu Chen
}

Issued: NOVEMBER 1996 


\section{ABSTRACT}

The health risks for an individual exposed to contaminants released from SRS outfalls from 1989 to 1995 were estimated. The exposure pathways studied are ingestion of drinking water, ingestion of contaminated fish and dermal contact with contaminants in water while swimming. The estimated incremental risks for an individual developing cancer vary from 3.E-06 to $1.0 \mathrm{E}-05$. The estimated total exposure chronic noncancer hazard indices vary from 6.E-02 to 1.E-01.

The critical contaminants were ranked based on their cancer risks and chronic noncarcinogenic hazard quotients. For cancer risks, the critical contaminants released from SRS outfalls are arsenic, tetrachloroethylene, and benzene. For chronic noncarcinogenic risks, the critical contaminants released from SRS outfalls are cadmium, arsenic, silver, chromium, mercury, selenium, nitrate, manganese, zinc, nickel, uranium, barium, copper, tetrachloroethylene, cyanide, and phenol.

The critical pathways in decreasing risk order are ingestion of contaminated fish, ingestion of drinking water and dermal contact with contaminants in water while swimming. 


\section{TABLE OF CONIENTS}

1. INTRODUCTION 1

2. CALCULATIONS 2

2.1 Source Term 3

2.2 Concentration at US Highway 301

2.3 Residential Exposure: Ingestion of Contaminants in Drinking Water

2.4 Residential Exposure: Ingestion of Contaminants in Fish 4

2.5 Residential Exposure: Dermal Exposure While Swimming 4

2.6 Carcinogenic Effects 5

2.7 Noncarcinogenic Effects 6

2.8 Aggregate Risks for Multiple Contaminants 6

2.9 Combining Risks Across Exposure Pathways 7

2.10 Ranking for Contaminants and Pathways 8

3. CONCLUSIONS 8

$\begin{array}{ll}\text { REFERENCES } & 10\end{array}$ 


\section{LIST OF TABLES}

Table 1 Contaminants Released from the Savannah River Site Outfalls

Table 1 (Continued) Contaminants Released from the Savannah River Site Outfalls

Table 1 (Continued) Contaminants Released from the Savannah River Site Outfalls

Table 1 (Continued) Contaminants Released from the Savannah River Site Outfalls

Table 1 (Continued) Contaminants Released from the Savannah River Site Outfalls

Table 1 (Continued) Contaminants Released from the Savannah River Site Outfalls

Table 2 Calculated Source Terms for Contaminants Released from SRS Outfalls

Table 3 Calculated Concentrations at US Highway 301

Table 4 Calculated Exposures from Ingestion of Carcinogenic Contaminants in Drinking Water

Table 5 Calculated Exposures from Ingestion of Noncarcinogenic Contaminants in Drinking Water

Table 6 Calculated Carcinogenic Contaminant Exposures from Ingestion of Contaminated Fish

Table 7 Calculated Noncarcinogenic Contaminant Exposures from Ingestion of Contaminated Fish

Table 8 Calculated Exposures from Dermal Contact with Carcinogenic Contaminants in Water While Swimming

Table 9 Calculated Exposures from Dermal Contact with Noncarcinogenic Contaminants in Water While Swimming 
Table 10 Contaminants' Toxicity

Table 11 Estimated Cancer Risk for Contaminants Released from SRS Outfalls

Table 11 (continued) Estimated Cancer Risk for Contaminants Released from SRS Outfalls

Table 11 (continued) Estimated Cancer Risk for Contaminants Released from SRS Outfalls

Table 11 (continued) Estimated Cancer Risk for Contaminants Released from SRS Outfalls

Table 12 Estimated Chronic Noncarcinogenic Hazard Quotients for Contaminants Released from SRS Outfalls

Table 12 (continued) Estimated Chronic Noncarcinogenic Hazard Quotients for Contaminants Released from SRS Outfalls 32

Table 12 (continued) Estimated Chronic Noncarcinogenic

Hazard Quotients for Contaminants Released from SRS

Outfalls

Table 12 (continued) Estimated Chronic Noncarcinogenic

Hazard Quotients for Contaminants Released from SRS

Outfalls .34

Table 13 Critical Contaminant Ranking Based on Total Exposure Cancer Risk

Table 14 Critical Contaminant Ranking Based on Total Exposure Hazard Quotient 


\title{
CRITICAL CONTAMINANT/CRITICAL PATHWAY ANALYSIS - SURFACE WATER TRANSPORT FOR NONRADIOACTIVE CONTAMINANTS (U)
}

\author{
Kuo-Fu Chen \\ Westinghouse Savannah River Company \\ Savannah River Site \\ Aiken, SC 29808
}

\section{INTRODUCTION}

A complete and thorough critical-contaminant/critical-pathway analysis for routine environmental releases is required by the Savannah River Site (SRS) Environmental Monitoring Plan Corrective Action Plan (ESH-EMS-94-0129). The results will serve as the rationale and design criteria for the SRS Environmental Monitoring Program. The Environmental Monitoring Section requested the Environmental Technology Section to perform the following tasks: 1) identify, evaluate, and compile Savannah River data, aqueous contaminant source terms, and river use data; 2) calculate historic doses, prospective doses, and risks associated with the doses; and 3) determine critical contaminants and critical pathways.

The contaminants released from SRS outfalls from 1989 to 1995 were compiled and the release rates ( $\mathrm{kg} /$ day) for these contaminants were calculated based on historical records measured by the SRS Environmental Protection Department.

The transport of the contaminants via the Savannah River from SRS to the US Highway 301 bridge were simulated by the WASP5 computer code $[1,2]$. WASP5 is a US Environmental Protection Agency (EPA) water quality analysis program that simulates pollutant transport through surface water. WASP5 uses a finite difference method to solve the advective transport equation. The concentrations of the contaminants at US Highway 301 were calculated.

The health risks for an individual exposed to contaminants released from SRS outfalls from 1989 to 1995, through various exposure pathways were estimated. The exposure pathways studied are ingestion of drinking water, ingestion of contaminated fish and dermal contact with contaminants in water while swimming. The estimated incremental risks for an individual developing cancer vary from 3.E-06 to $1.0 \mathrm{E}-05$. The estimated total exposure chronic noncancer hazard indices vary from 6.E-02 to 1.E-01. 
The critical contaminants were ranked based on their cancer risks and chronic noncarcinogenic hazard quotients. For cancer risks, the critical contaminants released from SRS outfalls are arsenic, tetrachloroethylene, and benzene. For chronic noncarcinogenic risks, the critical contaminants released from SRS outfalls are cadmium, arsenic, silver, chromium, mercury, selenium, nitrate, manganese, zinc, nickel, uranium, barium, copper, tetrachloroethylene, cyanide, and phenol.

The critical pathways in decreasing risk order are ingestion of contaminated fish, ingestion of drinking water and dermal contact with contaminants in water while swimming.

\section{CALCULATIONS}

The following procedure was used to estimate the health risks for an individual who consumes drinking water and fishes from the Savannah River, and swimming in the Savannah River downstream from SRS at the US Highway 301 bridge. These risks are resulted from exposures to the nonradioactive contaminants released from SRS outfalls.

1. Release rates (kg/day) of contaminants from SRS outfalls were compiled and quantified.

2. The total release rate for each contaminant released from SRS as a whole was calculated.

3. Contaminant transport via the Savannah River from SRS to the US Highway 301 bridge was simulated, and contaminant concentrations at this location were calculated.

4. Exposures from ingestion of water and fish, and swimming for each noncarcinogenic and carcinogenic contaminant were calculated.

5. The cancer risks from ingestion of drinking water, ingestion of contaminated fish and dermal contact while swimming for each contaminant were calculated.

6. The noncarcinogenic hazard quotients from ingestion of drinking water, ingestion of contaminated fish and dermal contact while swimming for each contaminant were calculated.

7. Aggregate cancer risks and noncancer hazard quotients for multiple contaminants were calculated.

8. Critical contaminants were ranked based on cancer risks and noncancer hazard quotients.

Detailed explanations of each calculation step in this procedure follow. 


\subsection{Source Term}

The release rate for contaminant $i$ from outfall $j$ is calculated as:

$$
p_{i}^{j}=u c_{i}^{j} Q^{j}
$$

where

$$
\begin{aligned}
& p_{i}^{j}=\text { Contaminant Release Rate, } \mathrm{kg} / \mathrm{day} \\
& c_{i}^{j}=\text { Contaminant Concentration, } \mathrm{mg} / \mathrm{l} \\
& Q^{j}=\text { Discharge Flow Rate, } 10^{6} \text { gallon } / \text { day } \\
& u=\text { Unit Conversion Factor }
\end{aligned}
$$

Table 1 lists the contaminants released from SRS outfalls. The historical records of the measured contaminant concentrations and the discharge flow rates were obtained from SRS Environmental Data reports [3 - 9].

The total release rate of contaminant $i$ from SRS is obtained by adding the release rates from each outfall:

$$
P_{i}=\sum_{j=1}^{n} p_{i}^{j}
$$

Table 2 shows the calculated total release rates (source terms) from SRS for each contaminant from 1989 to 1995.

\subsection{Concentration at US Highway 301}

With the contaminant release rate (source term) calculated by Equation 2 and the average of the Savannah River daily average flow measured at Augusta, Georgia gauge station for the year in study [10 - 16], WASP5 simulates the contaminant transport from SRS to US Highway 301 and calculates $C_{i}$, the contaminant concentration in the Savannah River at US Highway 301 . Table 3 presents the calculated concentrations.

\subsection{Residential Exposure: Ingestion of Contaminants in Drinking Water}

The residential average daily intake for ingestion of contaminant $i$ in drinking water is calculated by [17]:

$$
\mathrm{DI}_{i}=\frac{C_{i} \times \mathrm{IR} \times \mathrm{EF} \times \mathrm{ED}}{\mathrm{BW} \times \mathrm{AT}}
$$


where

$\mathrm{DI}_{i}=$ Daily intake (exposure) of Contaminant $i, \mathrm{mg} / \mathrm{kg}$-day

$C_{i}=$ Concentration of Contaminant $i$, Calculated by WASP5, mg $/$

$\mathrm{IR}=$ Ingestion Rate, 2 liters per day

$\mathrm{EF}=$ Exposure Frequency, 365 days per year

$\mathrm{ED}=$ Exposure Duration, 30 years

$\mathrm{BW}=$ Body Weight, $70 \mathrm{~kg}$

$\mathrm{AT}=$ Averaging Time (period over which exposure is averaged)

$=30$ year $\mathrm{x} 365$ day/year for noncarcinogenic effects

$=70$ year $\mathrm{x} 365$ day/year for carcinogenic effects

Tables 4 and 5 show the calculated exposures for ingestion of carcinogenic and noncarcinogenic contaminants in drinking water, respectively.

\subsection{Residential Exposure: Ingestion of Contaminants in Fish}

The residential average daily intake for ingestion of contaminant $i$ in contaminated fish is calculated by [18]:

$$
\mathrm{DI}_{i}=\frac{C_{i} \times \mathrm{BF} \times \mathrm{IR} \times \mathrm{FI} \times \mathrm{EF} \times \mathrm{ED}}{\mathrm{BW} \times \mathrm{AT}}
$$

where

$\mathrm{DI}_{i}=$ Daily intake (exposure) of Contaminant $i, \mathrm{mg} / \mathrm{kg}$-day

$C_{i}=$ Concentration of Contaminant $i$, Calculated by WASP5, mg $/$

$\mathrm{BF}=$ Bioaccumulation Factor, $1 / \mathrm{kg}$

$\mathrm{IR}=$ Ingestion Rate, $\mathrm{kg} / \mathrm{meal}$

$\mathrm{FI}=$ Fraction ingested (unitless)

$\mathrm{EF}=$ Exposure Frequency, meals/year

$\mathrm{ED}=$ Exposure Duration, 30 years

$\mathrm{BW}=$ Body Weight, $70 \mathrm{~kg}$

$\mathrm{AT}=$ Averaging Time (period over which exposure is averaged)

$=30$ year $\times 365$ day/year for noncarcinogenic effects

$=70$ year $\mathrm{x} 365$ day/year for carcinogenic effects

The bioaccumulation factor, $\mathrm{BF}$, is contaminant specific and is obtained from Reference 19. The product of ingestion rate, IR, and exposure frequency, $\mathrm{EF}$, is kilogram of fish consumed per year and is provided by Reference 20 as 19 $\mathrm{kg} /$ day. The fraction ingested, FI, is assumed be one. Tables 6 and 7 show the calculated exposures for ingestion of carcinogenic and noncarcinogenic contaminants in fish, respectively.

\subsection{Residential Exposure: Dermal Exposure While Swimming}

The residential exposure for dermal contact with contaminant $i$ in water while swimming is calculated by [18]: 


$$
\mathrm{DI}_{i}=\frac{C_{i} \times \mathrm{SA} \times \mathrm{K} \times \mathrm{ET} \times \mathrm{EF} \times \mathrm{ED} \times \mathrm{CF}}{\mathrm{BW} \times \mathrm{AT}}
$$

where

$$
\begin{aligned}
\mathrm{DI}_{i} & =\text { Daily intake (exposure) of Contaminant } i, \mathrm{mg} / \mathrm{kg} \text {-day } \\
C_{i} & =\text { Concentration of Contaminant } i, \text { Calculated by WASP5, mg/l } \\
\mathrm{SA} & =\text { Total body surface area, } \mathrm{m}^{2} \\
\mathrm{~K} & =\text { Permeability constant, } \mathrm{cm} / \mathrm{hr} \\
\mathrm{ET} & =\text { Exposure time, hr/day } \\
\mathrm{EF} & =\text { Exposure Frequency, day/year } \\
\mathrm{ED} & =\text { Exposure Duration, } 30 \text { years } \\
\mathrm{CF} & =\text { Conversion factor, } 10^{-3} \mathrm{l} / \mathrm{cm}^{3} \\
\mathrm{BW} & =\text { Body Weight, } 70 \mathrm{~kg} \\
\mathrm{AT} & =\text { Averaging Time (period over which exposure is averaged) } \\
& =30 \text { year } \times 365 \text { day/year for noncarcinogenic effects } \\
& =70 \text { year } \times 365 \text { day/year for carcinogenic effects }
\end{aligned}
$$

Reference 17 gives the total body surface area, $\mathrm{SA}$, of $1.94 \mathrm{~m}^{2}$ and the permeability constant, $\mathrm{K}$, of $1.5 \times 10^{-3} \mathrm{~cm} / \mathrm{hr}$. The product of exposure time, $\mathrm{ET}$, and the exposure frequency, EF, is the number of swimming hours per year and is $8.9 \mathrm{hr} / \mathrm{year}$ for site specific value [20]. Tables 8 and 9 show the calculated exposures for dermal contact of carcinogenic and noncarcinogenic contaminants while swimming, respectively.

Based on the intake or exposure calculated from Equations 3 to 5, the health risk for carcinogenic and noncarcinogenic effects associated with a particular contaminant can be determined.

\subsection{Carcinogenic Effect}

Risks for carcinogens are estimated as the incremental probability of an individual developing cancer over a lifetime as a result of exposure to the potential carcinogen. The slope factor (SF) converts estimated daily intakes averaged over a lifetime of exposure (given by Equations 3 to 5 ) to an incremental risk of an individual developing cancer. The linear low-dose cancer risk equation is

$$
\operatorname{Risk}_{i}=\mathrm{DI}_{i} \times \mathrm{SF}_{i}
$$

where

$$
\begin{aligned}
& \text { Risk }_{i}=\text { A unitless probability of an individual developing cancer } \\
& \mathrm{SF}_{i}=\text { Slope factor for contaminant } i(\mathrm{mg} / \mathrm{kg}-\text { day })^{-1} \\
& \mathrm{DI}_{i}=\text { Chronic daily intake averaged over } 70 \text { years, } \mathrm{mg} / \mathrm{kg} \text {-day }
\end{aligned}
$$


The slope factor shown in Table 10 for a particular contaminant is obtained from References 21 and 22 . Table 11 presents the calculated cancer risks for contaminants released from SRS outfalls from 1989 to 1995.

\subsection{Noncarcinogenic Effects}

Currently, the EPA does not use a probabilistic approach to estimate the potential for noncarcinogenic health effects. Instead, the potential for noncarcinogenic effects is evaluated by comparing an exposure level (intake) over a specified time period (e.g., a lifetime) with a reference dose derived for a similar exposure period [17]. This ratio of exposure to toxicity is called a hazard quotient and is expressed as:

$$
\mathrm{NHQ}_{i}=\frac{\mathrm{DI}_{i}}{\mathrm{RfD}_{i}}
$$

where

$\mathrm{NHQ}_{i}=$ Noncancer Hazard Quotient for Exposure of contaminant $i$

$\mathrm{DI}_{i}=$ Daily intake (exposure level) of contaminant $i$

$\mathrm{RfD}_{i}=$ Reference dose for contaminant $i$

$\mathrm{DI}_{i}$ and $\mathrm{RfD}_{i}$ are expressed in the same units and represent the same chronic exposure period. The reference dose (RfD) for a particular contaminant is obtained from References 21 and 22 .

The noncancer hazard quotient assumes that there is a level of intake or exposure (i.e., RfD) below which it is unlikely for even sensitive populations to experience adverse health effects. If the intake level (DI) exceeds this threshold (i.e., if NHQ exceeds unity), there may be concerns for potential noncancer effects. Table 12 shows the calculated chronic noncarcinogenic hazard quotients for contaminants released from SRS outfalls from 1989 to 1995.

\subsection{Aggregate Risks for Multiple Contaminants}

Multiple contaminants are released from SRS outfalls. To assess the overall potential for cancer and noncancer effects posed by multiple contaminants, EPA [23] has developed guidelines for the health risk assessment of chemical mixtures.

The total incremental individual lifetime cancer risk for simultaneous exposure to several carcinogens is approximated by summation of the risks for all the contaminants

$$
\operatorname{Risk}_{T}=\sum_{i=1}^{m} \operatorname{Risk}_{i}
$$


where

$$
\begin{aligned}
& \operatorname{Risk}_{T}=\text { Total cancer risk } \\
& \operatorname{Risk}_{i}=\text { Cancer risk for contaminant } i
\end{aligned}
$$

Table 11 presents the calculated total cancer risks for simultaneous exposure to contaminants released from SRS outfalls from 1989 to 1995.

To assess the overall potential for noncarcinogenic effects posed by multiple contaminant intakes (Exposures), a hazard index (HI) approach has been developed based on EPA's Guidelines for Health Risk Assessment of Chemical Mixtures. This approach assumes that simultaneous subthreshold exposures to several contaminants could result in an adverse health effect. It also assumes that the magnitude of the adverse health effect will be proportional to the sum of the hazard quotients.

$$
\mathrm{HI}=\sum_{i=1}^{m} \mathrm{NHQ}_{i}
$$

where

$\mathrm{HI}=$ Chronic noncarcinogenic hazard index for exposure to multiple contaminants $\mathrm{NHQ}_{i}=$ Chronic noncarcinogenic hazard quotient for contaminant $i$

Table 12 shows the calculated chronic noncarcinogenic hazard indices for simultaneous exposure to contaminants released from SRS outfalls from 1989 to 1995 .

\subsection{Combining Risks Across Exposure Pathways}

EPA [17] assumes that the total cancer risks from various exposure pathways are approximated to be additive, as long as the risks are for the same individual and time period. The cancer risk equation for multiple pathways is expressed as:

$$
\text { Total Exposure Cancer Risk }=\sum_{i} \operatorname{Risk}_{(\text {pathway })_{i}}
$$

Table 11 presents the calculated total exposure cancer risks for multiple exposure pathways to contaminants released from SRS outfalls from 1989 to 1995.

The hazard index equation for multiple pathways is given by Reference 17 as: 


$$
\begin{aligned}
& \text { Total Chronic Noncarcinogenic Exposure Hazard Index }= \\
& \sum_{i} \text { Hazard Index }_{(\text {pathway })_{i}}
\end{aligned}
$$

Table 12 shows the calculated total chronic noncarcinogenic hazard index for multiple exposure pathways to contaminants released from SRS outfalls from 1989 to 1995.

\subsection{Ranking for Contaminants and Pathways}

Tables 13 and 14 show the ranking of the critical contaminants released from SRS from 1989 to 1995 . The ranking is based on the cancer risk and the chronic noncarcinogenic hazard quotient. For cancer risks, the critical contaminants released from SRS outfalls are arsenic, tetrachloroethylene, and benzene. For noncarcinogenic risks, the critical contaminants released from SRS outfalls are cadmium, arsenic, silver, chromium, mercury, selenium, nitrate, manganese, zinc, nickel, uranium, barium, copper, tetrachloroethylene, cyanide, and phenol.

Ingestion of contaminated fish poses higher risk than drinking water and swimming because the contaminants tend to accumulate in the tissue of the fish. Swimming poses the least risk to an individual because fewer contaminants are permeated through the skin.

\section{CONCLUSIONS}

The health risks for an individual exposed to contaminants released from SRS outfalls from 1989 to 1995 through various exposure pathways were estimated. The exposure pathways studied are ingestion of drinking water, ingestion of contaminated fish and dermal contact with contaminants in water while swimming. The estimated total incremental lifetime exposure cancer risks vary from 3.E-06 to $1.0 \mathrm{E}-05$. The estimated total exposure chronic noncancer hazard indices vary from 6.E-02 to 1.E-01.

The contaminants released from SRS outfalls were ranked. The ranking is based on the cancer risk and the chronic noncarcinogenic hazard quotient. For cancer risks, the critical contaminants released from SRS outfalls are arsenic, tetrachloroethylene, and benzene. For noncarcinogenic risks, the critical contaminants released from SRS outfalls are cadmium, arsenic, silver, chromium, mercury, selenium, nitrate, manganese, zinc, nickel, uranium, barium, copper, tetrachloroethylene, cyanide, and phenol.

The calculated chronic noncancer hazard quotients for dermal contact with contaminants in water while swimming vary from $4 \mathrm{E}-06$ to $1 \mathrm{E}-05$, for ingestion of drinking water vary from $1 \mathrm{E}-02$ to $3 \mathrm{E}-02$, for ingestion of fish vary from $5 \mathrm{E}-02$ to $8 \mathrm{E}-02$. 
The calculated cancer risks for dermal contact with contaminants in water while swimming vary from $3 \mathrm{E}-10$ to $1 \mathrm{E}-09$, for ingestion of drinking water vary from 7E-07 to $3 \mathrm{E}-06$, for ingestion of fish vary from $2 \mathrm{E}-06$ to $8 \mathrm{E}-06$. 


\section{REFERENCES}

1. Ambrose, R. B., Wool, T. A., and Martin, J. L., "The Water Quality Analysis Simulation Program, WASP5, Part A: Model Documentation; Part B: Input Dataset," Environmental Research Laboratory, Office of Research and Development, U.S. Environmental Protection Agency, Athens, Georgia, September 20, 1993.

2. Chen, Kuo-Fu, "Revised STREAM Code and WASP5 Benchmark," WSRCRP-95-598, May 1995.

3. Cummins, C. L., Martin, D. K., and Todd, J. L., "SRS Environmental Data for 1989," WSRC-IM-90-60, Volume II, Figures and Data Tables.

4. Cummins, C. L., Martin, D. K., and Todd, J. L., "SRS Environmental Data for 1990," WSRC-IM-91-28, Volume I, Environmental Monitoring, Compliance, and Research. \& Summary tamphizt only

5. Arnett, M. W., Karapatakis, L. K., Mamtey, A. R., Todd, J. L., "SRS Environmental Report for 1991," WSRC-TR-92-186.

$\checkmark$ 6. Arnett, M. W., "SRS Environmental Report for 1992," WSRC-TR-93-077.

7. Arnett, M. W., "SRS Environmental Report for 1993," WSRC-TR-94-077.

$\checkmark$ 8. Arnett, M. W., "SRS Environmental Report for 1994," WSRC-TR-95-077.

9. Arnett, M. W., "SRS Environmental Report for 1995," WSRC-TR-96-0077.

10. Bennett, C. S., Cooney, T. W., Jones, K. H., Church, B. W., and Murray, G. L., "Water Resources Data South Carolina, Water Year 1989," USGS Water-Data Report SC-89-1.

11. Bennett, C. S., Cooney, T. W., Jones, K. H., Conrads, P. A., "Water Resources Data South Carolina, Water Year 1990," USGS Water-Data Report SC-90-1.

12. Bennett, C. S., Cooney, T. W., Jones, K. H., Conrads, P. A., "Water Resources Data South Carolina, Water Year 1991," USGS Water-Data Report SC-91-1.

of 13. Bennett, C. S., Cooney, T. W., Jones, K. H., Gissendanner, J. W., "Water Resources Data South Carolina, Water Year 1992," USGS Water-Data Report SC-92-1.

14. Bennett, C. S., Cooney, T. W., Jones, K. H., Drewes, P. A. "Water Resources Data South Carolina, Water Year 1993," USGS Water-Data Report SC-93-1. 
k 15. Cooney, T. W., Jones, K. H., Drewes, P. A., Gissendanner, J. W., and Church, B. W., "Water Resources Data South Carolina, Water Year 1994," USGS Water-Data Report SC-94-1

of 16. Flow for 1995 provided by Frank Melandez from USGS New Ellenton Field Office.

siz 17. "Risk Assessment Guidance for Superfund Volume I Human Health Evaluation Manual (Part A) Interim Final," EPA/540/1-89/002. December 1989.

18. "Plant-Wide Basline Human Health Risk Assessment, Portsmouth Gaseous Diffusion Plant, Piketon, Ohio," Volime 1, DOE/OR/111403/V1\&D1, POEF-ER-4625/V1\&D1, August 29, 1995.

19. Thompson, Stanley E., Burton, C. Ann, Quinn, Dorothy J. and Ng, Yook C., "Concentration Factors of Chemical Elements in Edible Aquatic Organisms," UCRL-50564 Rev. 1, TID-4500, UC-48, October 10, 1972.

20. Hamby, David M., "Land and Water Use Characteristics in the Vicinity of the Savannah River Site (U)," WSRC-RP-91-17, March 1991.

di.. 21. "Health Effects Assessment Summary Tables, FY-1995 Annual," EPA/540/R-95/036, May 1995.

ok 22. "Electronic Handbook of Risk Assessment Values", Volume 5, Issue 5, March 15, 1996.

of 23. "Guideline for the Health Risk Assessment of Chemical Mixtures," 51 Federal Register 34014, September 24,1986. 


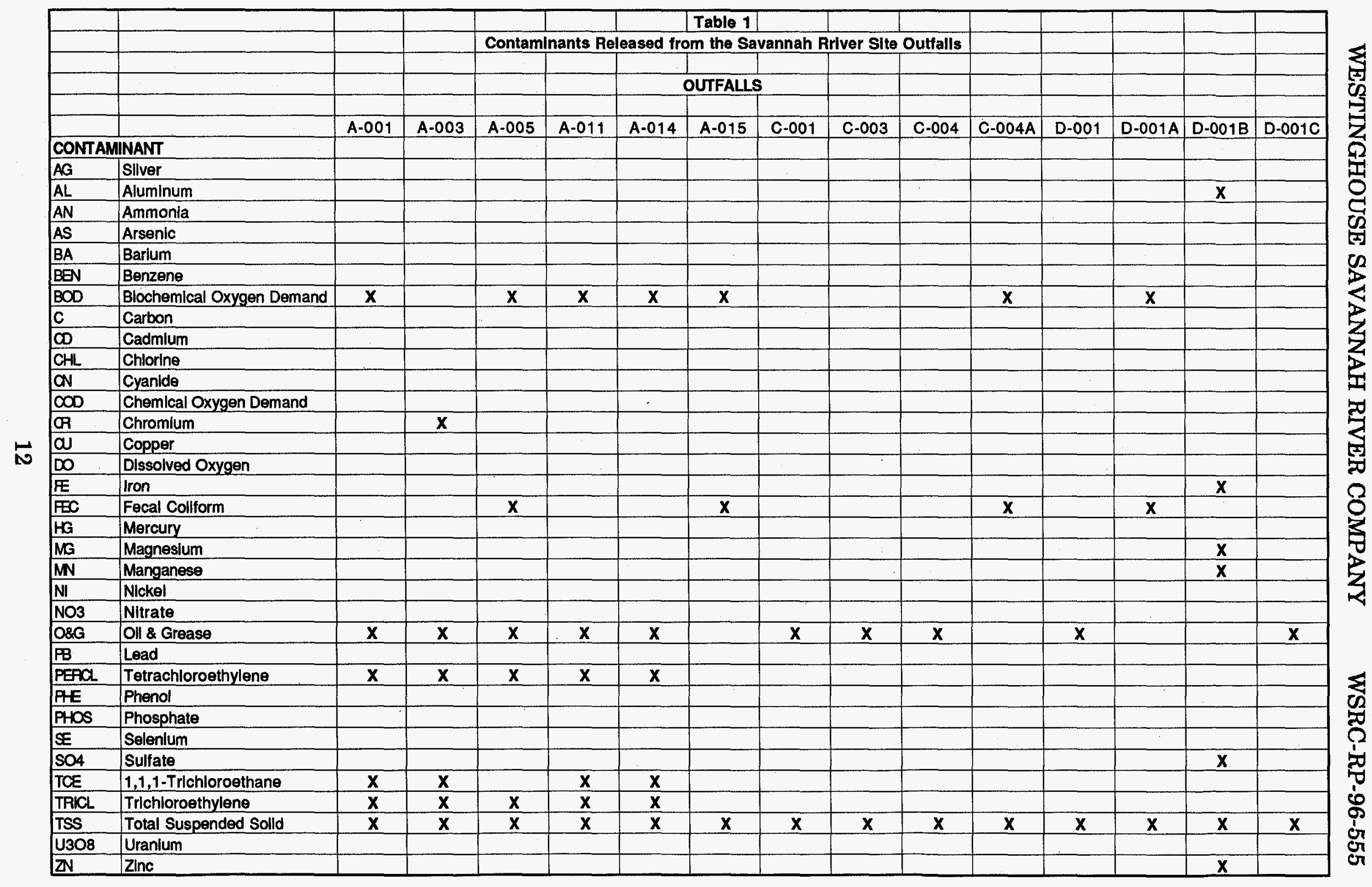




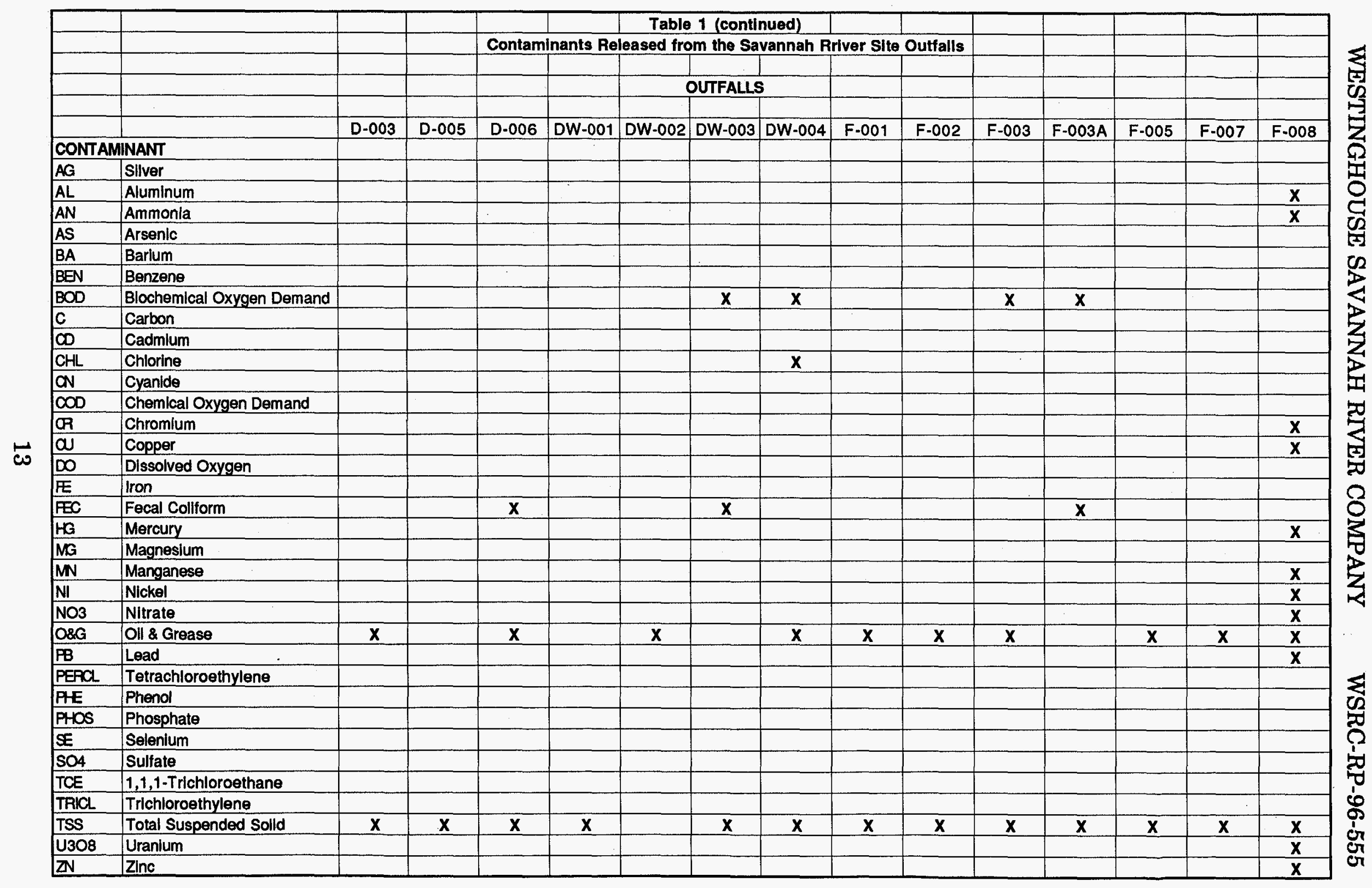




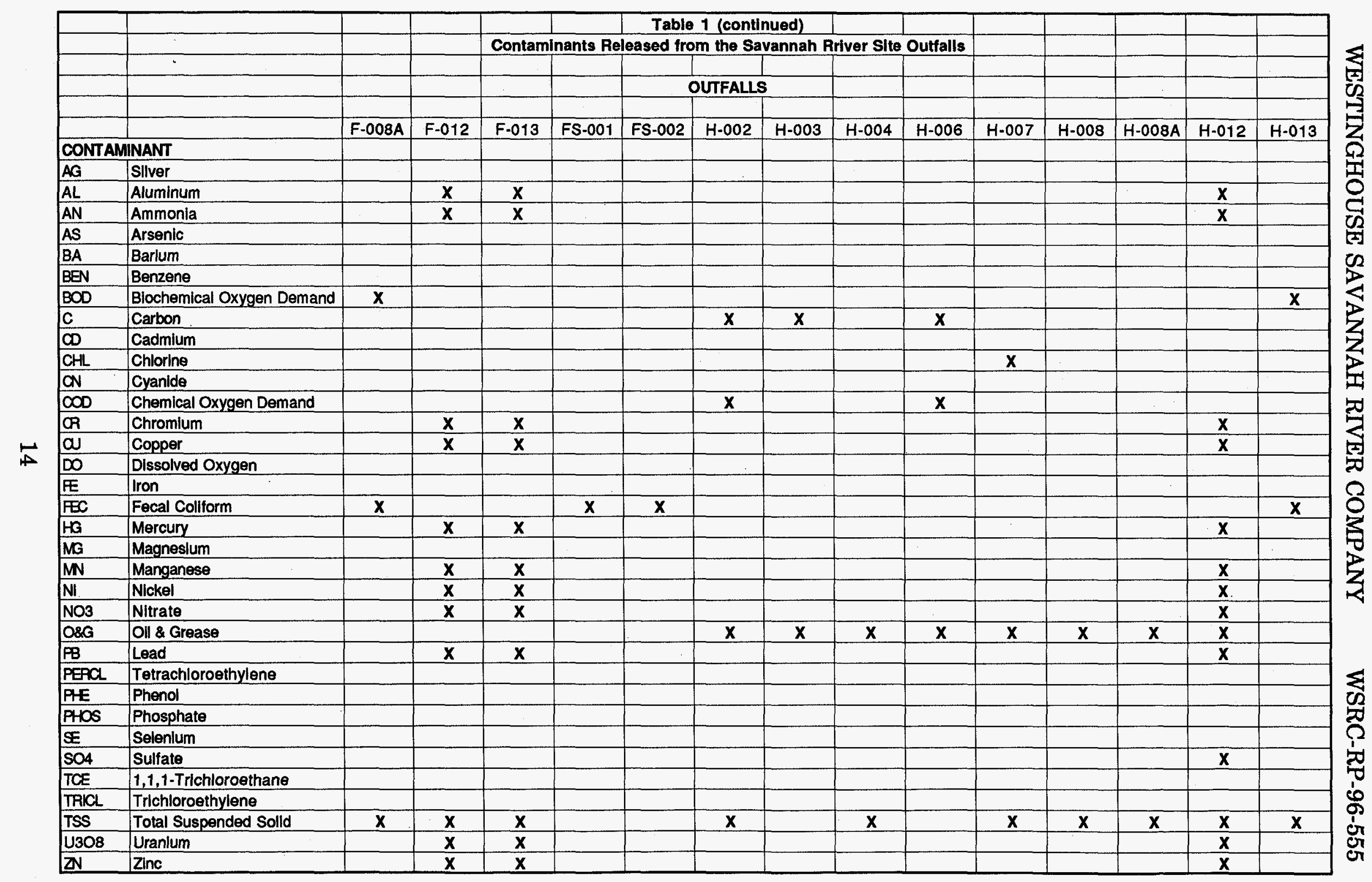




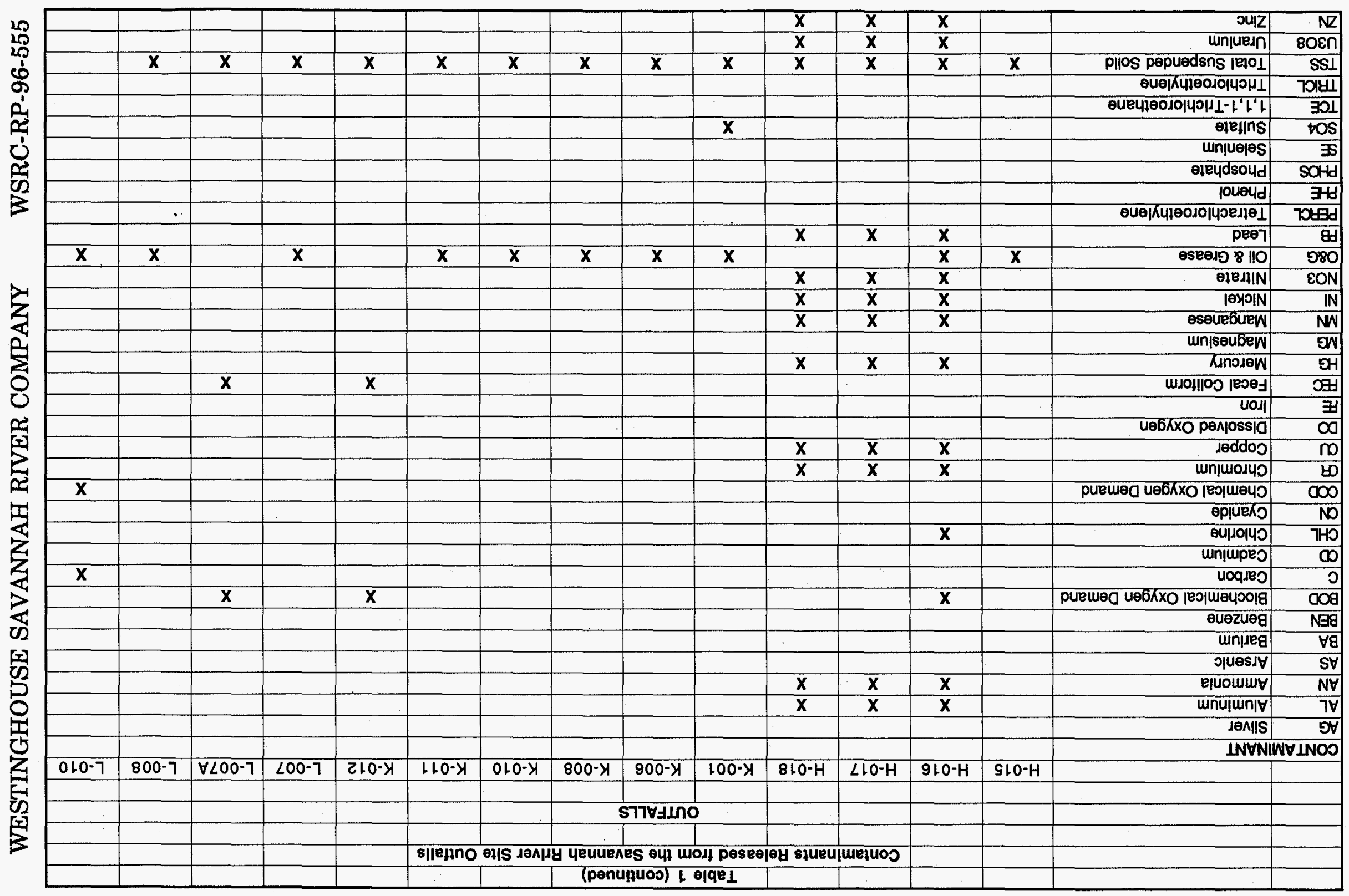




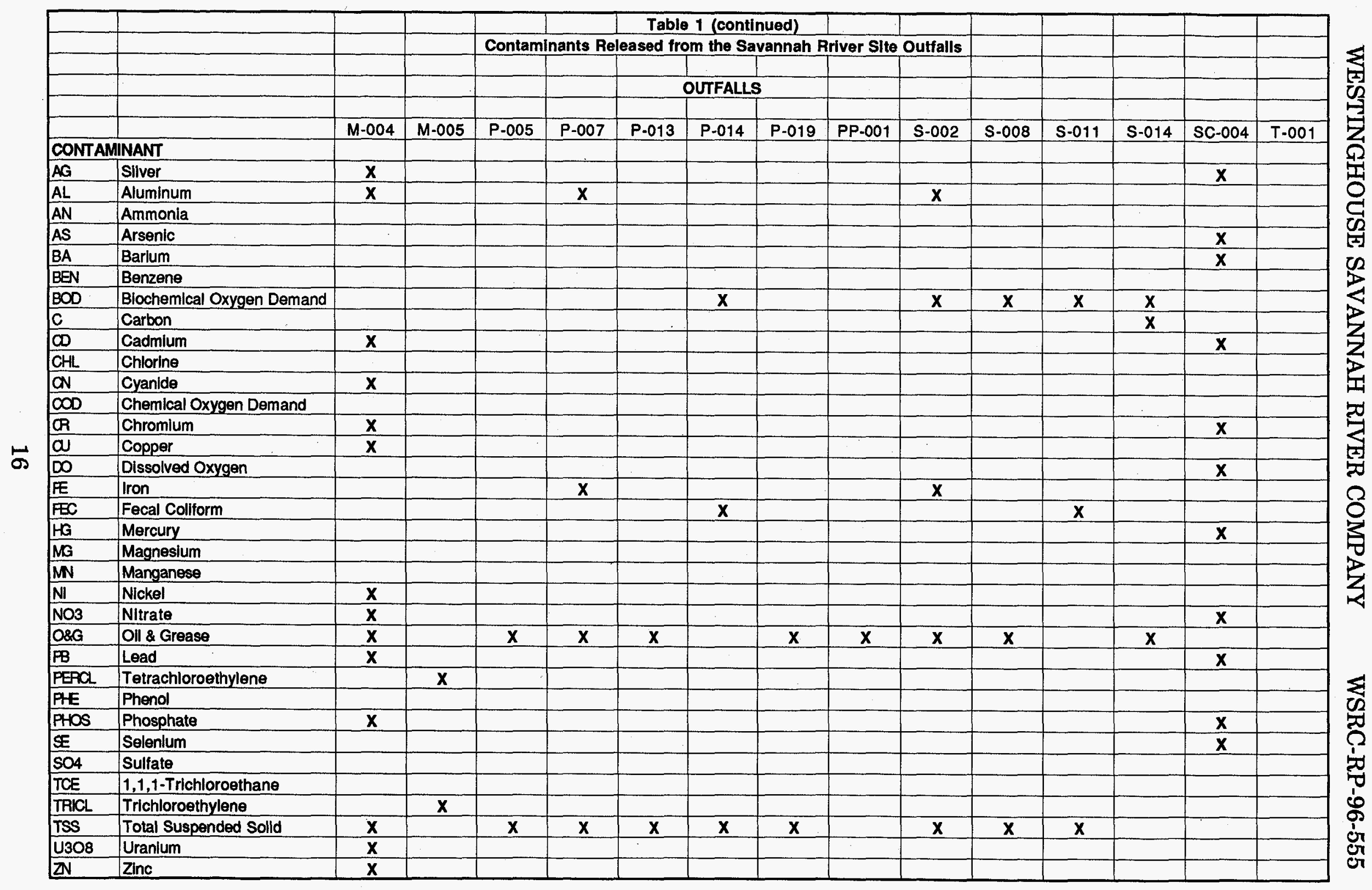




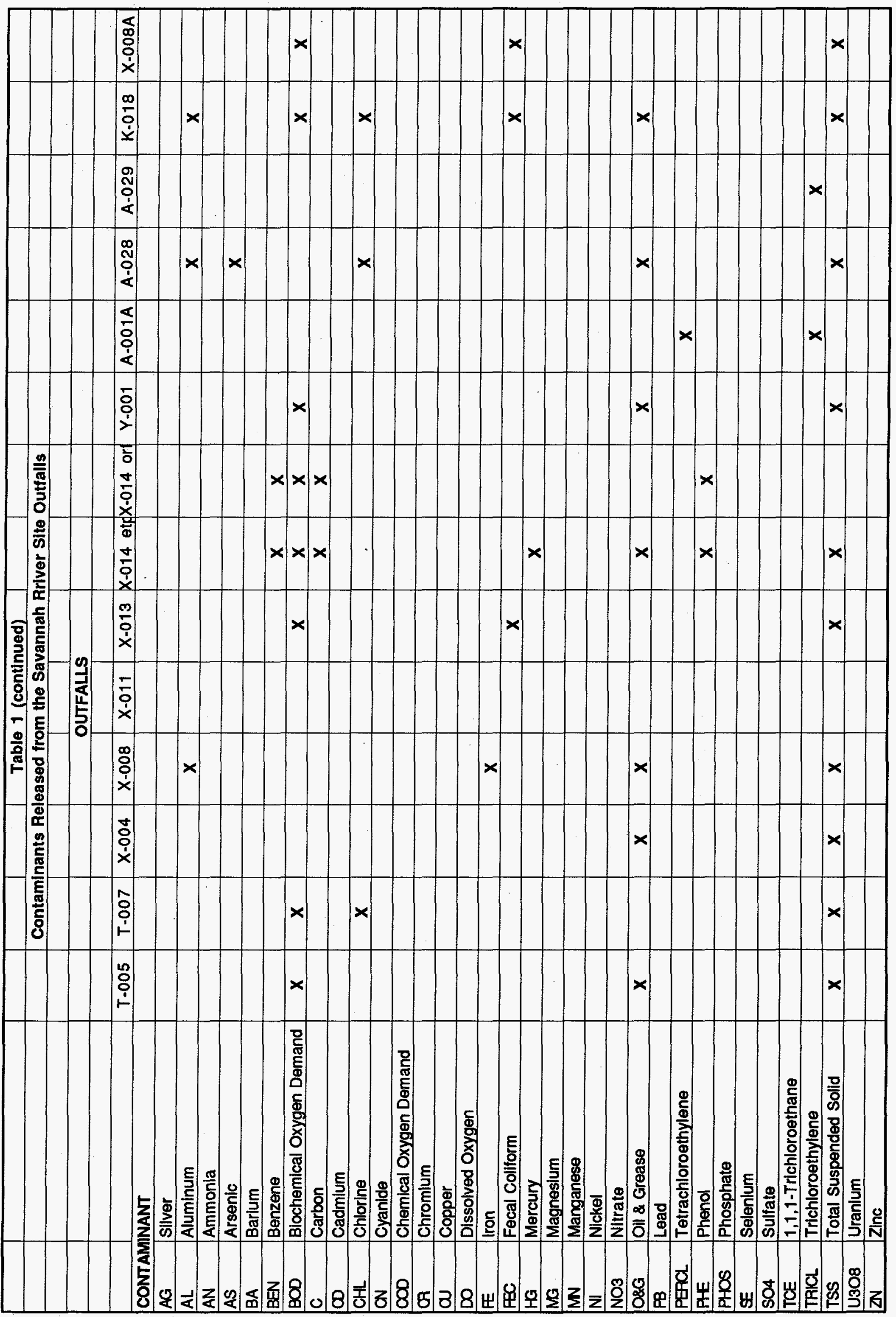


Table 2

Calculated Source Terms for Contaminants Released from SRS Outfalls

\begin{tabular}{|c|c|c|c|c|c|c|c|c|}
\hline & Contaminant & $\begin{array}{c}1989 \\
\mathrm{~kg} / \text { day }\end{array}$ & $\begin{array}{c}1990 \\
\mathrm{~kg} / \text { day }\end{array}$ & $\begin{array}{c}1991 \\
\mathrm{~kg} / \text { day }\end{array}$ & $\begin{array}{c}1992 \\
\mathrm{~kg} / \text { day }\end{array}$ & $\begin{array}{c}1993 \\
\mathrm{~kg} / \text { day }\end{array}$ & $\begin{array}{c}1994 \\
\mathrm{~kg} / \text { day }\end{array}$ & $\begin{array}{c}1995 \\
\mathrm{~kg} / \text { day }\end{array}$ \\
\hline $\mathrm{AG}$ & Silver & $1.53 \mathrm{E}-01$ & 4.15E-01 & $1.57 \mathrm{E}-01$ & $1.26 \mathrm{E}-01$ & $6.45 E+01$ & $1.02 \mathrm{E}-01$ & $6.05 \mathrm{E}+01$ \\
\hline $\mathrm{AL}$ & Aluminum & $6.54 \mathrm{E}+00$ & $8.93 E+00$ & $8.15 E+00$ & $3.84 \mathrm{E}+00$ & $2.99 \mathrm{E}+01$ & $2.59 \mathrm{E}+01$ & $4.56 \mathrm{E}+01$ \\
\hline AN & Ammonia & $1.31 \mathrm{E}+00$ & $1.08 \mathrm{E}+00$ & $3.43 E+00$ & $5.57 \mathrm{E}+00$ & $2.95 E+00$ & $3.24 \mathrm{E}+00$ & $7.50 \mathrm{E}+00$ \\
\hline $\mathrm{AS}$ & Arsenic & $9.15 \mathrm{E}-01$ & $7.78 \mathrm{E}-01$ & $9.42 \mathrm{E}-01$ & $7.56 \mathrm{E}-01$ & $1.72 \mathrm{E}+00$ & $1.02 \mathrm{E}+00$ & $3.66 \mathrm{E}+00$ \\
\hline BA & Barium & $4.79 E+00$ & $3.21 E+00$ & $3.93 E+00$ & $2.75 E+00$ & $4.38 \mathrm{E}+00$ & $2.70 \mathrm{E}+00$ & $2.45 \mathrm{E}+00$ \\
\hline BEN & Benzene & $3.86 \mathrm{E}-04$ & $3.77 \mathrm{E}-05$ & $1.59 \mathrm{E}-04$ & 4.62E-05 & $5.00 \mathrm{E}-05$ & $2.12 \mathrm{E}-05$ & $8.52 \mathrm{E}-05$ \\
\hline $\mathrm{C}$ & Carbon & $5.61 \mathrm{E}+00$ & $9.26 \mathrm{E}-01$ & $1.08 \mathrm{E}+00$ & $7.20 \mathrm{E}-01$ & $1.16 \mathrm{E}+00$ & $6.54 \mathrm{E}-01$ & $3.24 \mathrm{E}-01$ \\
\hline $\mathrm{CD}$ & Cadmium & $3.05 \mathrm{E}+00$ & $2.59 \mathrm{E}+00$ & $3.14 \mathrm{E}+00$ & $2.52 \mathrm{E}+00$ & $3.45 \mathrm{E}+00$ & $2.05 E+00$ & $9.16 \mathrm{E}-01$ \\
\hline $\mathrm{CHL}$ & Chlorine & $2.92 \mathrm{E}-01$ & $2.12 \mathrm{E}-01$ & $2.55 \mathrm{E}-01$ & 2.83E-01 & $9.89 \mathrm{E}+00$ & $1.10 \mathrm{E}+01$ & $9.15 \mathrm{E}+00$ \\
\hline $\mathrm{CN}$ & Cyanide & $4.94 \mathrm{E}-04$ & $1.71 \mathrm{E}-04$ & $1.17 \mathrm{E}-04$ & $2.69 \mathrm{E}-04$ & $3.73 \mathrm{E}-04$ & $2.16 \mathrm{E}-04$ & $6.06 \mathrm{E}-04$ \\
\hline CR & Chromium & $8.48 E+00$ & $5.70 E+00$ & $6.71 \mathrm{E}+00$ & $5.43 E+00$ & $1.09 \mathrm{E}+01$ & $4.80 \mathrm{E}+00$ & $2.07 E+00$ \\
\hline $\mathrm{CU}$ & Copper & $4.10 \mathrm{E}-01$ & $5.08 \mathrm{E}-01$ & $2.20 \mathrm{E}-01$ & $3.81 \mathrm{E}-01$ & $3.14 \mathrm{E}-01$ & $2.38 \mathrm{E}-01$ & $2.73 \mathrm{E}-01$ \\
\hline $\mathrm{FE}$ & Iron & $2.52 E+00$ & $8.17 \mathrm{E}+00$ & $7.57 \mathrm{E}+00$ & $1.24 \mathrm{E}+00$ & $1.58 \mathrm{E}+01$ & $2.26 \mathrm{E}+00$ & $1.01 \mathrm{E}+02$ \\
\hline $\mathrm{HG}$ & Merc & $3.28 \mathrm{E}-02$ & $1.08 \mathrm{E}-01$ & $6.57 \mathrm{E}-02$ & $3.86 \mathrm{E}-02$ & $1.51 \mathrm{E}-01$ & $3.11 \mathrm{E}-02$ & $5.77 \mathrm{E}-02$ \\
\hline $\mathrm{MN}$ & Manganese & $2.92 \mathrm{E}-01$ & 5.33E-01 & $4.87 \mathrm{E}-01$ & $3.40 \mathrm{E}-01$ & 5.07E-01 & $3.36 \mathrm{E}-01$ & $3.21 \mathrm{E}+00$ \\
\hline NI & Nickel & $1.13 \mathrm{E}+00$ & $1.26 \mathrm{E}+00$ & $1.03 E+00$ & $4.73 E-01$ & $8.80 \mathrm{E}-01$ & $7.59 \mathrm{E}-01$ & $1.92 \mathrm{E}-01$ \\
\hline NO3 & Nitrate & $1.20 \mathrm{E}+02$ & $7.01 \mathrm{E}+01$ & $6.42 \mathrm{E}+01$ & $9.01 \mathrm{E}+01$ & $1.31 E+02$ & $1.23 \mathrm{E}+02$ & $1.06 \mathrm{E}+02$ \\
\hline O\&G & Oil \& Grease & $2.12 E+03$ & $1.23 \mathrm{E}+03$ & $3.33 \mathrm{E}+03$ & $3.20 \mathrm{E}+03$ & $9.32 \mathrm{E}+02$ & $1.48 \mathrm{E}+03$ & $8.25 E+02$ \\
\hline PB & Lead & $2.17 \mathrm{E}+00$ & $2.51 \mathrm{E}+00$ & $2.17 \mathrm{E}+00$ & $1.21 \mathrm{E}+00$ & $8.42 \mathrm{E}-01$ & $5.32 \mathrm{E}+00$ & $3.89 \mathrm{E}+00$ \\
\hline PERCL & Tetrachloroethylene & $2.04 \mathrm{E}-02$ & $2.00 \mathrm{E}-02$ & $2.03 \mathrm{E}-02$ & $2.54 \mathrm{E}-02$ & 4.07E-02 & $2.39 \mathrm{E}-02$ & $6.20 \mathrm{E}-02$ \\
\hline PHE & Phenol & $1.10 \mathrm{E}-03$ & $1.78 \mathrm{E}-04$ & $3.76 \mathrm{E}-04$ & $2.31 \mathrm{E}-04$ & $9.99 \mathrm{E}-05$ & $1.19 E-04$ & $4.07 \mathrm{E}-04$ \\
\hline PHOS & Phosphate & $3.83 E+01$ & $1.70 \mathrm{E}+01$ & $3.82 \mathrm{E}+01$ & $8.27 E+00$ & $1.08 \mathrm{E}+01$ & $7.53 E+00$ & $2.68 \mathrm{E}+01$ \\
\hline SE & Selenium & $1.83 \mathrm{E}+00$ & $1.56 \mathrm{E}+00$ & $1.88 \mathrm{E}+00$ & $1.51 \mathrm{E}+00$ & $1.72 E+00$ & $1.23 \mathrm{E}+00$ & $3.66 \mathrm{E}+00$ \\
\hline SO4 & Sulfate & $1.80 \mathrm{E}+03$ & $3.03 \mathrm{E}+02$ & $8.67 E+01$ & $2.12 \mathrm{E}+01$ & $1.00 \mathrm{E}+02$ & $2.66 \mathrm{E}+01$ & $4.74 \mathrm{E}+02$ \\
\hline TCE & 1,1,1-Trichloroethane & $1.74 \mathrm{E}-02$ & $1.42 \mathrm{E}-02$ & $1.40 \mathrm{E}-02$ & $1.79 \mathrm{E}-02$ & $1.68 \mathrm{E}-02$ & $1.28 \mathrm{E}-02$ & 4.79E-02 \\
\hline TRICL & Trichloroethylene & $5.59 \mathrm{E}-02$ & $2.94 \mathrm{E}-02$ & $2.03 \mathrm{E}-02$ & $3.19 \mathrm{E}-02$ & 4.85E-02 & $1.03 \mathrm{E}-01$ & $5.85 \mathrm{E}-02$ \\
\hline $\mathrm{U} 308$ & Uranium & $5.73 \mathrm{E}-01$ & $1.12 \mathrm{E}+00$ & $2.70 \mathrm{E}-01$ & $3.85 \mathrm{E}-01$ & $1.22 E+00$ & $4.15 \mathrm{E}-01$ & $1.18 \mathrm{E}+01$ \\
\hline $\mathrm{ZN}$ & Zinc & $1.30 \mathrm{E}+00$ & $2.77 \mathrm{E}+00$ & $1.60 \mathrm{E}+00$ & $1.67 \mathrm{E}+00$ & $2.01 E+00$ & $1.90 \mathrm{E}+00$ & $2.78 \mathrm{E}+00$ \\
\hline
\end{tabular}


Table 3

Calculated Concentrations at US Highway 301

\begin{tabular}{cc} 
& 1989 \\
Contaminant & $\mathrm{mg} / \mathrm{l}$ \\
& \\
AG & $8.719 \mathrm{E}-06$ \\
AL & $3.737 \mathrm{E}-04$ \\
AN & $7.498 \mathrm{E}-05$ \\
AS & $5.230 \mathrm{E}-05$ \\
BA & $2.737 \mathrm{E}-04$ \\
BEN & $2.229 \mathrm{E}-08$ \\
C & $3.204 \mathrm{E}-04$ \\
CD & $1.744 \mathrm{E}-04$ \\
CHL & $1.671 \mathrm{E}-05$ \\
CN & $2.800 \mathrm{E}-08$ \\
CR & $4.844 \mathrm{E}-04$ \\
CU & $2.343 \mathrm{E}-05$ \\
FE & $1.440 \mathrm{E}-04$ \\
HG & $1.874 \mathrm{E}-06$ \\
MN & $1.669 \mathrm{E}-05$ \\
NI & $6.452 \mathrm{E}-05$ \\
NO3 & $6.878 \mathrm{E}-03$ \\
O\&G & $1.211 \mathrm{E}-01$ \\
PB & $1.241 \mathrm{E}-04$ \\
PERCL & $1.164 \mathrm{E}-06$ \\
PHE & $6.286 \mathrm{E}-08$ \\
PHOS & $2.188 \mathrm{E}-03$ \\
SE & $1.046 \mathrm{E}-04$ \\
SO4 & $1.026 \mathrm{E}-01$ \\
TCE & $9.949 \mathrm{E}-07$ \\
TRICL & $3.195 \mathrm{E}-06$ \\
U3O8 & $3.273 \mathrm{E}-05$ \\
ZN & $7.442 \mathrm{E}-05$ \\
& \\
\hline
\end{tabular}

1991

$\mathrm{mg} /$

mg/

1.596E-05

3.435E-04

4.141E-05

2.992E-05

1.237E-04

1.538E-09

3.561E-05

9.973E-05

8.152E-06

6.539E-09

2.194E-04

3.141E-04

4.163E-06

2.051E-05

4.859E-05

2.698E-03

4.724E-02

9.652E-05

7.712E-07

6.923E-09

6.556E-04

5.983E-05

1.167E-02

5.446E-07

1.130E-06

4.309E-05

1.067E-04
1.955E-05
6.417E-06

3.330E-04

1.399E-04

3.850E-05

1.604E-04

6.536E-09

4.421E-05

1.283E-04

1.043E-05

4.902E-09

2.740E-04

8.994E-06

3.094E-04

2.682E-06

1.990E-05

6.547E-05

2.623E-03

1.359E-01

8.878E-05

8.292E-07

1.552E-08

1.559E-03

7.700E-05

3.541E-03

5.719E-07

8.292E-07

1.104E-05

7.762E-05
1992

$\mathrm{mg} / \mathrm{l}$

4.900E-06

1.494E-04

2.166E-04

2.939E-05

1.068E-04

1.945E-09

2.799E-05

9.799E-05

1.100E-05

1.050E-08

2.114E-04

1.483E-05

4.810E-05

1.502E-06

1.323E-05

1.841E-05

3.503E-03

1.245E-01

4.706E-05

9.888E-07

8.946E-09

3.218E-04

5.878E-05

8.248E-04

6.951E-07

1.242E-06

$1.498 \mathrm{E}-05$

6.492E-05

\section{3}

mg/l

2.168E-03

1.005E-03

9.903E-05

5.797E-05

1.472E-04

1.680E-09

3.906E-05

1.161E-04

3.325E-04

1.244E-08

3.656E-04

1.055E-05

5.315E-04

5.084E-06

1.704E-05

2.959E-05

4.404E-03

3.133E-02

2.829E-05

1.368E-06

3.361E-09

3.621E-04

5.797E-05

3.374E-03

5.636E-07

1.631E-06

4.085E-05

6.742E-05
1994

mg/l

1995

mg/

4.106E-06

1.038E-03

1.297E-04

4.105E-05

1.084E-04

8.019E-10

2.622E-05

8.227E-05

4.416E-04

8.821E-09

1.925E-04

9.527E-06

9.044E-05

1.248E-06

1.346E-05

$3.045 \mathrm{E}-05$

$4.920 \mathrm{E}-03$

5.939E-02

2.133E-04

9.590E-07

4.811E-09

3.019E-04

4.926E-05

$1.068 \mathrm{E}-03$

5.128E-07

4.136E-06

1.664E-05

7.627E-05
2.191E-03

1.652E-03

2.718E-04

1.328E-04

8.896E-05

3.261E-09

1.172E-05

3.320E-05

3.316E-04

2.210E-08

7.511E-05

9.899E-06

$3.657 \mathrm{E}-03$

2.091E-06

$1.165 \mathrm{E}-04$

6.963E-06

3.839E-03

2.989E-02

1.408E-04

2.248E-06

1.486E-08

9.694E-04

1.328E-04

1.719E-02

1.735E-06

2.118E-06

4.277E-04

1.006E-04 
Table 4

Calculated Exposures from Ingestion of Carcinogenic Contaminants in Drinking Water

\begin{tabular}{|c|c|c|c|c|c|c|c|}
\hline Contaminant & $\begin{array}{c}1989 \\
\mathrm{mg} / \mathrm{kg} \text {-day }\end{array}$ & $\begin{array}{c}1990 \\
\mathrm{mg} / \mathrm{kg} \text {-day }\end{array}$ & $\begin{array}{c}1991 \\
\mathrm{mg} / \mathrm{kg} \text {-day }\end{array}$ & $\begin{array}{c}1992 \\
\mathrm{mg} / \mathrm{kg} \text {-day }\end{array}$ & $\begin{array}{c}1993 \\
\mathrm{mg} / \mathrm{kg} \text {-day }\end{array}$ & $\begin{array}{c}1994 \\
\mathrm{mg} / \mathrm{kg} \text {-day }\end{array}$ & $\begin{array}{c}1995 \\
\mathrm{mg} / \mathrm{kg}-\text { day }\end{array}$ \\
\hline $\mathrm{AG}$ & $1.07 \mathrm{E}-07$ & $1.95 \mathrm{E}-07$ & $7.86 \mathrm{E}-08$ & $6.00 \mathrm{E}-08$ & $2.65 \mathrm{E}-05$ & $5.03 \mathrm{E}-08$ & $2.68 \mathrm{E}-05$ \\
\hline $\mathrm{AL}$ & $4.58 \mathrm{E}-06$ & $4.21 \mathrm{E}-06$ & $4.08 \mathrm{E}-06$ & $1.83 \mathrm{E}-06$ & 1.23E-05 & $1.27 \mathrm{E}-05$ & $2.02 \mathrm{E}-05$ \\
\hline AN & $9.18 \mathrm{E}-07$ & $5.07 \mathrm{E}-07$ & $1.71 \mathrm{E}-06$ & $2.65 \mathrm{E}-06$ & $1.21 \mathrm{E}-06$ & $1.59 \mathrm{E}-06$ & $3.33 \mathrm{E}-06$ \\
\hline AS & $6.40 \mathrm{E}-07$ & $3.66 \mathrm{E}-07$ & $4.71 \mathrm{E}-07$ & $3.60 \mathrm{E}-07$ & $7.10 \mathrm{E}-07$ & 5.03E-07 & $1.63 \mathrm{E}-06$ \\
\hline BA & $3.35 \mathrm{E}-06$ & $1.51 \mathrm{E}-06$ & $1.96 \mathrm{E}-06$ & $1.31 \mathrm{E}-06$ & $1.80 \mathrm{E}-06$ & $1.33 \mathrm{E}-06$ & $1.09 \mathrm{E}-06$ \\
\hline BEN & $2.73 \mathrm{E}-10$ & $1.88 \mathrm{E}-11$ & $8.00 \mathrm{E}-11$ & $2.38 \mathrm{E}-11$ & $2.06 \mathrm{E}-11$ & $9.82 \mathrm{E}-12$ & $3.99 \mathrm{E}-11$ \\
\hline C & $3.92 \mathrm{E}-06$ & $4.36 \mathrm{E}-07$ & $5.41 \mathrm{E}-07$ & 3.43E-07 & $4.78 \mathrm{E}-07$ & $3.21 \mathrm{E}-07$ & $1.44 \mathrm{E}-07$ \\
\hline CD & 2.14E-06 & $1.22 \mathrm{E}-06$ & $1.57 \mathrm{E}-06$ & $1.20 \mathrm{E}-06$ & $1.42 \mathrm{E}-06$ & $1.01 \mathrm{E}-06$ & $4.07 \mathrm{E}-07$ \\
\hline CHL & $2.05 \mathrm{E}-07$ & $9.98 \mathrm{E}-08$ & $1.28 \mathrm{E}-07$ & $1.35 \mathrm{E}-07$ & 4.07E-06 & $5.41 \mathrm{E}-06$ & $4.06 \mathrm{E}-06$ \\
\hline $\mathrm{CN}$ & $3.43 \mathrm{E}-10$ & $8.01 \mathrm{E}-11$ & $6.00 \mathrm{E}-11$ & $1.29 \mathrm{E}-10$ & $1.52 \mathrm{E}-10$ & $1.08 \mathrm{E}-10$ & $2.71 \mathrm{E}-10$ \\
\hline CR & $5.93 \mathrm{E}-06$ & $2.69 \mathrm{E}-06$ & $3.36 \mathrm{E}-06$ & 2.59E-06 & $4.48 \mathrm{E}-06$ & $2.36 \mathrm{E}-06$ & $9.20 \mathrm{E}-07$ \\
\hline CU & 2.87E-07 & $2.39 \mathrm{E}-07$ & $1.10 \mathrm{E}-07$ & $1.82 \mathrm{E}-07$ & $1.29 \mathrm{E}-07$ & $1.17 \mathrm{E}-07$ & $1.21 \mathrm{E}-07$ \\
\hline $\mathrm{FE}$ & $1.76 \mathrm{E}-06$ & $3.85 \mathrm{E}-06$ & $3.79 \mathrm{E}-06$ & $5.89 \mathrm{E}-07$ & $6.51 \mathrm{E}-06$ & $1.11 \mathrm{E}-06$ & $4.48 \mathrm{E}-05$ \\
\hline $\mathrm{HG}$ & $2.29 \mathrm{E}-08$ & $5.10 \mathrm{E}-08$ & $3.28 \mathrm{E}-08$ & $1.84 \mathrm{E}-08$ & $6.23 \mathrm{E}-08$ & $1.53 \mathrm{E}-08$ & $2.56 \mathrm{E}-08$ \\
\hline MN & $2.04 \mathrm{E}-07$ & 2.51E-07 & $2.44 \mathrm{E}-07$ & $1.62 \mathrm{E}-07$ & 2.09E-07 & $1.65 \mathrm{E}-07$ & $1.43 \mathrm{E}-06$ \\
\hline NI & $7.90 \mathrm{E}-07$ & $5.95 \mathrm{E}-07$ & $8.02 \mathrm{E}-07$ & $2.25 \mathrm{E}-07$ & 3.62E-07 & $3.73 \mathrm{E}-07$ & $8.53 \mathrm{E}-08$ \\
\hline NO3 & 8.42E-05 & $3.30 \mathrm{E}-05$ & $3.21 \mathrm{E}-05$ & $4.29 \mathrm{E}-05$ & 5.39E-05 & $6.02 \mathrm{E}-05$ & $4.70 \mathrm{E}-05$ \\
\hline O\&G & $1.48 \mathrm{E}-03$ & 5.78E-04 & $1.66 \mathrm{E}-03$ & $1.52 \mathrm{E}-03$ & 3.84E-04 & $7.27 \mathrm{E}-04$ & $3.66 \mathrm{E}-04$ \\
\hline PB & $1.52 \mathrm{E}-06$ & $1.18 \mathrm{E}-06$ & $1.09 \mathrm{E}-06$ & $5.76 \mathrm{E}-07$ & $3.46 \mathrm{E}-07$ & $2.61 \mathrm{E}-06$ & $1.72 \mathrm{E}-06$ \\
\hline PERCL & $1.43 \mathrm{E}-08$ & $9.44 \mathrm{E}-09$ & $1.02 \mathrm{E}-08$ & $1.21 \mathrm{E}-08$ & $1.68 \mathrm{E}-08$ & $1.17 \mathrm{E}-08$ & $2.75 \mathrm{E}-08$ \\
\hline PHE & $7.70 \mathrm{E}-10$ & $8.48 \mathrm{E}-11$ & $1.90 \mathrm{E}-10$ & $1.10 \mathrm{E}-10$ & $4.12 \mathrm{E}-11$ & $5.89 \mathrm{E}-11$ & $1.82 \mathrm{E}-10$ \\
\hline PHOS & $2.68 \mathrm{E}-05$ & $8.03 \mathrm{E}-06$ & $1.91 \mathrm{E}-05$ & $3.94 \mathrm{E}-06$ & 4.43E-06 & $3.70 \mathrm{E}-06$ & $1.19 \mathrm{E}-05$ \\
\hline $\mathrm{SE}$ & $1.28 \mathrm{E}-06$ & 7.33E-07 & $9.43 \mathrm{E}-07$ & $7.20 \mathrm{E}-07$ & 7.10E-07 & $6.03 \mathrm{E}-07$ & $1.63 \mathrm{E}-06$ \\
\hline SO4 & $1.26 \mathrm{E}-03$ & $1.43 \mathrm{E}-04$ & $4.34 \mathrm{E}-05$ & $1.01 \mathrm{E}-05$ & $4.13 \mathrm{E}-05$ & $1.31 \mathrm{E}-05$ & $2.10 \mathrm{E}-04$ \\
\hline TCE & $1.22 \mathrm{E}-08$ & 6.67E-09 & $7.00 \mathrm{E}-09$ & 8.51E-09 & $6.90 \mathrm{E}-09$ & $6.28 \mathrm{E}-09$ & $2.12 \mathrm{E}-08$ \\
\hline TRICL & $3.91 \mathrm{E}-08$ & $1.38 \mathrm{E}-08$ & $1.02 \mathrm{E}-08$ & $1.52 \mathrm{E}-08$ & $2.00 \mathrm{E}-08$ & $5.06 \mathrm{E}-08$ & $2.59 \mathrm{E}-08$ \\
\hline U308 & $4.01 \mathrm{E}-07$ & $5.28 \mathrm{E}-07$ & $1.35 \mathrm{E}-07$ & $1.83 \mathrm{E}-07$ & $5.00 \mathrm{E}-07$ & $2.04 \mathrm{E}-07$ & $5.24 \mathrm{E}-06$ \\
\hline ZN & $9.11 \mathrm{E}-07$ & $1.31 \mathrm{E}-06$ & $9.50 \mathrm{E}-07$ & 7.95E-07 & 8.26E-07 & 9.34E-07 & $1.23 \mathrm{E}-06$ \\
\hline
\end{tabular}


Table 5

Calculated Exposures from Ingestion of Noncarcinogenic Contaminants in Drinking Water

\begin{tabular}{|c|c|c|c|c|c|c|c|}
\hline Contaminant & $\begin{array}{c}1989 \\
\mathrm{mg} / \mathrm{kg}-\text { day }\end{array}$ & $\begin{array}{c}1990 \\
\mathrm{mg} / \mathrm{kg}-\text { day }\end{array}$ & $\begin{array}{c}1991 \\
\mathrm{mg} / \mathrm{kg}-\text { day }\end{array}$ & $\begin{array}{c}1992 \\
\text { mg/kg-day }\end{array}$ & $\begin{array}{c}1993 \\
\mathrm{mg} / \mathrm{kg}-\mathrm{day}\end{array}$ & $\begin{array}{c}1994 \\
\mathrm{mg} / \mathrm{kg}-\mathrm{day}\end{array}$ & $\begin{array}{c}1995 \\
\mathrm{mg} / \mathrm{kg}-\text { day }\end{array}$ \\
\hline $\mathrm{AG}$ & 2.49E-07 & $4.56 \mathrm{E}-07$ & $1.83 \mathrm{E}-07$ & $1.40 \mathrm{E}-07$ & $6.19 \mathrm{E}-05$ & 1.17E-07 & 6.26E-05 \\
\hline $\mathrm{AL}$ & 1.07E-05 & 9.81E-06 & 9.51E-06 & $4.27 \mathrm{E}-06$ & $2.87 \mathrm{E}-05$ & 2.97E-05 & $4.72 E-05$ \\
\hline $\mathrm{AN}$ & 2.14E-06 & $1.18 \mathrm{E}-06$ & 4.00E-06 & $6.19 \mathrm{E}-06$ & 2.83E-06 & $3.71 \mathrm{E}-06$ & 7.77E-06 \\
\hline AS & $1.49 \mathrm{E}-06$ & $8.55 \mathrm{E}-07$ & 1.10E-06 & 8.40E-07 & $1.66 \mathrm{E}-06$ & 1.17E-06 & $3.79 \mathrm{E}-06$ \\
\hline BA & 7.82E-06 & 3.53E-06 & 4.58E-06 & 3.05E-06 & 4.21E-06 & $3.10 \mathrm{E}-06$ & 2.54E-06 \\
\hline BEN & 6.37E-10 & $4.39 \mathrm{E}-11$ & $1.87 \mathrm{E}-10$ & $5.56 \mathrm{E}-11$ & $4.80 \mathrm{E}-11$ & $2.29 \mathrm{E}-11$ & 9.32E-11 \\
\hline $\mathrm{C}$ & $9.15 \mathrm{E}-06$ & $1.02 \mathrm{E}-06$ & $1.26 \mathrm{E}-06$ & 8.00E-07 & $1.12 \mathrm{E}-06$ & $7.49 \mathrm{E}-07$ & $3.35 \mathrm{E}-07$ \\
\hline $\mathrm{CD}$ & $4.98 \mathrm{E}-06$ & $2.85 \mathrm{E}-06$ & 3.67E-06 & 2.80E-06 & $3.32 \mathrm{E}-06$ & 2.35E-06 & 9.49E-07 \\
\hline $\mathrm{CHL}$ & 4.77E-07 & 2.33E-07 & 2.98E-07 & 3.14E-07 & $9.50 \mathrm{E}-06$ & $1.26 \mathrm{E}-05$ & $9.47 \mathrm{E}-06$ \\
\hline $\mathrm{CN}$ & $8.00 \mathrm{E}-10$ & $1.87 \mathrm{E}-10$ & $1.40 \mathrm{E}-10$ & $3.00 \mathrm{E}-10$ & $3.55 \mathrm{E}-10$ & 2.52E-10 & $6.31 \mathrm{E}-10$ \\
\hline CR & $1.38 \mathrm{E}-05$ & 6.27E-06 & 7.83E-06 & 6.04E-06 & $1.04 \mathrm{E}-05$ & 5.50E-06 & 2.15E-06 \\
\hline $\mathrm{CU}$ & $6.69 \mathrm{E}-07$ & 5.59E-07 & 2.57E-07 & 4.24E-07 & 3.01E-07 & 2.72E-07 & 2.83E-07 \\
\hline FE & 4.11E-06 & 8.97E-06 & 8.84E-06 & 1.37E-06 & 1.52E-05 & $2.58 \mathrm{E}-06$ & $1.04 \mathrm{E}-04$ \\
\hline HG & 5.35E-08 & $1.19 \mathrm{E}-07$ & 7.66E-08 & 4.29E-08 & $1.45 \mathrm{E}-07$ & 3.57E-08 & 5.97E-08 \\
\hline $\mathrm{MN}$ & 4.77E-07 & $5.86 \mathrm{E}-07$ & 5.69E-07 & 3.78E-07 & 4.87E-07 & 3.85E-07 & $3.33 \mathrm{E}-06$ \\
\hline $\mathrm{NI}$ & $1.84 \mathrm{E}-06$ & $1.39 \mathrm{E}-06$ & $1.87 \mathrm{E}-06$ & $5.26 \mathrm{E}-07$ & 8.45E-07 & 8.70E-07 & $1.99 \mathrm{E}-07$ \\
\hline NO3 & 1.97E-04 & 7.71E-05 & $7.49 \mathrm{E}-05$ & $1.00 \mathrm{E}-04$ & $1.26 \mathrm{E}-04$ & $1.41 \mathrm{E}-04$ & 1.10E-04 \\
\hline O\&G & $3.46 \mathrm{E}-03$ & $1.35 \mathrm{E}-03$ & $3.88 \mathrm{E}-03$ & 3.56E-03 & 8.95E-04 & $1.70 \mathrm{E}-03$ & 8.54E-04 \\
\hline PB & $3.55 \mathrm{E}-06$ & $2.76 \mathrm{E}-06$ & 2.54E-06 & $1.34 \mathrm{E}-06$ & 8.08E-07 & $6.09 \mathrm{E}-06$ & 4.02E-0 \\
\hline PERCL & 3.33E-08 & 2.20E-08 & 2.37E-08 & 2.83E-08 & 3.91E-08 & 2.74E-08 & $6.42 \mathrm{E}-08$ \\
\hline PHE & 1.80E-09 & $1.98 \mathrm{E}-10$ & $4.43 \mathrm{E}-10$ & $2.56 \mathrm{E}-10$ & $9.60 \mathrm{E}-11$ & $1.37 \mathrm{E}-10$ & $4.25 \mathrm{E}-1 \mathrm{C}$ \\
\hline PHOS & $6.25 \mathrm{E}-05$ & 1.87E-05 & $4.45 \mathrm{E}-05$ & 9.19E-06 & $1.03 \mathrm{E}-05$ & 8.63E-06 & 2.77E-05 \\
\hline SE & 2.99E-06 & 1.71E-06 & $2.20 \mathrm{E}-06$ & 1.68E-06 & 1.66E-06 & $1.41 \mathrm{E}-06$ & 3.79E-06 \\
\hline SO4 & 2.93E-03 & 3.33E-04 & 1.01E-04 & 2.36E-05 & 9.64E-05 & 3.05E-05 & 4.91E-04 \\
\hline TCE & 2.84E-08 & $1.56 \mathrm{E}-08$ & $1.63 \mathrm{E}-08$ & $1.99 \mathrm{E}-08$ & $1.61 \mathrm{E}-08$ & $1.47 \mathrm{E}-08$ & 4.96E-08 \\
\hline TRICL & $9.13 \mathrm{E}-08$ & 3.23E-08 & 2.37E-08 & 3.55E-08 & $4.66 \mathrm{E}-08$ & $1.18 \mathrm{E}-07$ & $6.05 \mathrm{E}-08$ \\
\hline U308 & $9.35 \mathrm{E}-07$ & $1.23 \mathrm{E}-06$ & $3.15 \mathrm{E}-07$ & 4.28E-07 & 1.17E-06 & 4.75E-07 & 1.22E-05 \\
\hline $\mathrm{ZN}$ & 2.13E-06 & $3.05 \mathrm{E}-06$ & 2.22E-06 & $1.85 \mathrm{E}-06$ & $1.93 \mathrm{E}-06$ & 2.18E-06 & 2.87E-06 \\
\hline
\end{tabular}


Table 6

Calculated Exposures to Carcinogenic Contaminants from Ingestion of Contaminated Fish

N

$$
\begin{array}{lllllll}
1989 & 1990 & 1991 & 1992 & 1993 & 1994 & 1995
\end{array}
$$

Contaminant $\mathrm{mg} / \mathrm{kg}$-day $\mathrm{mg} / \mathrm{kg}$-day $\mathrm{mg} / \mathrm{kg}$-day $\mathrm{mg} / \mathrm{kg}$-day $\mathrm{mg} / \mathrm{kg}$-day $\mathrm{mg} / \mathrm{kg}$-day $\mathrm{mg} / \mathrm{kg}$-day

$\begin{array}{cccccccc}\text { AG } & 2.01 \mathrm{E}-05 & 1.17 \mathrm{E}-08 & 4.72 \mathrm{E}-09 & 3.61 \mathrm{E}-09 & 1.60 \mathrm{E}-06 & 3.02 \mathrm{E}-09 & 1.61 \mathrm{E}-06 \\ \text { AL } & 3.74 \mathrm{E}-03 & 1.09 \mathrm{E}-06 & 1.06 \mathrm{E}-06 & 4.76 \mathrm{E}-07 & 3.20 \mathrm{E}-06 & 3.31 \mathrm{E}-06 & 5.26 \mathrm{E}-06 \\ \text { AN } & & & & & & & \\ \text { AS } & 5.23 \mathrm{E}-03 & 9.54 \mathrm{E}-07 & 1.23 \mathrm{E}-06 & 9.37 \mathrm{E}-07 & 1.85 \mathrm{E}-06 & 1.31 \mathrm{E}-06 & 4.23 \mathrm{E}-06 \\ \text { BA } & 1.09 \mathrm{E}-03 & 1.58 \mathrm{E}-07 & 2.04 \mathrm{E}-07 & 1.36 \mathrm{E}-07 & 1.88 \mathrm{E}-07 & 1.38 \mathrm{E}-07 & 1.13 \mathrm{E}-07 \\ \text { BEN } & & & & & & & \\ \text { C } & 1.46 \mathrm{E}+00 & 5.16 \mathrm{E}-05 & 6.41 \mathrm{E}-05 & 4.06 \mathrm{E}-05 & 5.66 \mathrm{E}-05 & 3.80 \mathrm{E}-05 & 1.70 \mathrm{E}-05 \\ \text { CD } & 3.49 \mathrm{E}-02 & 6.36 \mathrm{E}-06 & 8.18 \mathrm{E}-06 & 6.25 \mathrm{E}-06 & 7.40 \mathrm{E}-06 & 5.24 \mathrm{E}-06 & 2.12 \mathrm{E}-06 \\ \text { CHL } & 8.36 \mathrm{E}-04 & 1.30 \mathrm{E}-07 & 1.66 \mathrm{E}-07 & 1.75 \mathrm{E}-07 & 5.30 \mathrm{E}-06 & 7.04 \mathrm{E}-06 & 5.28 \mathrm{E}-06 \\ \text { CN } & & & & & & & \\ \text { CR } & 9.69 \mathrm{E}-02 & 1.40 \mathrm{E}-05 & 1.75 \mathrm{E}-05 & 1.35 \mathrm{E}-05 & 2.33 \mathrm{E}-05 & 1.23 \mathrm{E}-05 & 4.79 \mathrm{E}-06 \\ \text { CU } & 1.17 \mathrm{E}-03 & 3.12 \mathrm{E}-07 & 1.43 \mathrm{E}-07 & 2.36 \mathrm{E}-07 & 1.68 \mathrm{E}-07 & 1.52 \mathrm{E}-07 & 1.58 \mathrm{E}-07 \\ \text { FE } & 1.44 \mathrm{E}-02 & 1.00 \mathrm{E}-05 & 9.86 \mathrm{E}-06 & 1.53 \mathrm{E}-06 & 1.69 \mathrm{E}-05 & 2.88 \mathrm{E}-06 & 1.17 \mathrm{E}-04 \\ \text { HG } & 1.87 \mathrm{E}-03 & 1.33 \mathrm{E}-06 & 8.55 \mathrm{E}-07 & 4.79 \mathrm{E}-07 & 1.62 \mathrm{E}-06 & 3.98 \mathrm{E}-07 & 6.66 \mathrm{E}-07 \\ \text { MN } & 6.68 \mathrm{E}-03 & 2.61 \mathrm{E}-06 & 2.54 \mathrm{E}-06 & 1.69 \mathrm{E}-06 & 2.17 \mathrm{E}-06 & 1.72 \mathrm{E}-06 & 1.49 \mathrm{E}-05 \\ \text { NI } & 6.45 \mathrm{E}-03 & 1.55 \mathrm{E}-06 & 2.09 \mathrm{E}-06 & 5.87 \mathrm{E}-07 & 9.43 \mathrm{E}-07 & 9.70 \mathrm{E}-07 & 2.22 \mathrm{E}-07 \\ \text { NO3 } & & & & & & & \\ \text { O\&G } & & & & & & & \\ \text { PB } & 1.24 \mathrm{E}-02 & 3.08 \mathrm{E}-06 & 2.83 \mathrm{E}-06 & 1.50 \mathrm{E}-06 & 9.02 \mathrm{E}-07 & 6.80 \mathrm{E}-06 & 4.49 \mathrm{E}-06 \\ \text { PERCL } & & & & & & & \\ \text { PHE } & & & & & & & \\ \text { PHOS } & 2.19 \mathrm{E}+02 & 2.09 \mathrm{E}-02 & 4.97 \mathrm{E}-02 & 1.03 \mathrm{E}-02 & 1.15 \mathrm{E}-02 & 9.62 \mathrm{E}-03 & 3.09 \mathrm{E}-02 \\ \text { SE } & 1.75 \mathrm{E}-02 & 3.18 \mathrm{E}-06 & 4.10 \mathrm{E}-06 & 3.13 \mathrm{E}-06 & 3.09 \mathrm{E}-06 & 2.62 \mathrm{E}-06 & 7.07 \mathrm{E}-06 \\ \text { SO4 } & & & & & & & \\ \text { TCE } & & & & & & & \\ \text { TRICL } & & & & & & & \\ \text { U3O8 } & 6.55 \mathrm{E}-05 & 2.75 \mathrm{E}-08 & 7.04 \mathrm{E}-09 & 9.55 \mathrm{E}-09 & 2.60 \mathrm{E}-08 & 1.06 \mathrm{E}-08 & 2.73 \mathrm{E}-07 \\ \text { ZN } & 1.49 \mathrm{E}-01 & 6.80 \mathrm{E}-05 & 4.95 \mathrm{E}-05 & 4.14 \mathrm{E}-05 & 4.30 \mathrm{E}-05 & 4.86 \mathrm{E}-05 & 6.41 \mathrm{E}-05\end{array}$


Table 7

Calculated Exposures to Noncarcinogenic Contaminants from Ingestion of Contaminated Fish

$\begin{array}{cccccccc}\text { Contaminant } & \begin{array}{c}1989 \\ \text { mg/kg-day }\end{array} & \begin{array}{c}1990 \\ \text { mg/kg-day }\end{array} & \begin{array}{c}1991 \\ \text { mg/kg-day }\end{array} & \begin{array}{c}1992 \\ \text { mg/kg-day }\end{array} & \begin{array}{c}1993 \\ \text { mg/kg-day }\end{array} & \begin{array}{c}1994 \\ \text { mg/kg-day }\end{array} & \begin{array}{c}1995 \\ \text { mg/kg-day }\end{array} \\ \text { AG } & 1.50 \mathrm{E}-08 & 2.74 \mathrm{E}-08 & 1.10 \mathrm{E}-08 & 8.42 \mathrm{E}-09 & 3.72 \mathrm{E}-06 & 7.05 \mathrm{E}-09 & 3.76 \mathrm{E}-06 \\ \text { AL } & 2.78 \mathrm{E}-06 & 2.55 \mathrm{E}-06 & 2.48 \mathrm{E}-06 & 1.11 \mathrm{E}-06 & 7.47 \mathrm{E}-06 & 7.72 \mathrm{E}-06 & 1.23 \mathrm{E}-05 \\ \text { AN } & & & & & & \\ \text { AS } & 3.89 \mathrm{E}-06 & 2.22 \mathrm{E}-06 & 2.86 \mathrm{E}-06 & 2.19 \mathrm{E}-06 & 4.31 \mathrm{E}-06 & 3.05 \mathrm{E}-06 & 9.88 \mathrm{E}-06 \\ \text { BA } & 8.14 \mathrm{E}-07 & 3.68 \mathrm{E}-07 & 4.77 \mathrm{E}-07 & 3.18 \mathrm{E}-07 & 4.38 \mathrm{E}-07 & 3.22 \mathrm{E}-07 & 2.65 \mathrm{E}-07 \\ \text { BEN } & & & & & & & \\ \text { C } & 1.08 \mathrm{E}-03 & 1.20 \mathrm{E}-04 & 1.50 \mathrm{E}-04 & 9.47 \mathrm{E}-05 & 1.32 \mathrm{E}-04 & 8.87 \mathrm{E}-05 & 3.97 \mathrm{E}-05 \\ \text { CD } & 2.59 \mathrm{E}-05 & 1.48 \mathrm{E}-05 & 1.91 \mathrm{E}-05 & 1.46 \mathrm{E}-05 & 1.73 \mathrm{E}-05 & 1.22 \mathrm{E}-05 & 4.94 \mathrm{E}-06 \\ \text { CHL } & 6.21 \mathrm{E}-07 & 3.03 \mathrm{E}-07 & 3.88 \mathrm{E}-07 & 4.09 \mathrm{E}-07 & 1.24 \mathrm{E}-05 & 1.64 \mathrm{E}-05 & 1.23 \mathrm{E}-05 \\ \text { CN } & & & & & & & \\ \text { CR } & 7.20 \mathrm{E}-05 & 3.26 \mathrm{E}-05 & 4.08 \mathrm{E}-05 & 3.14 \mathrm{E}-05 & 5.44 \mathrm{E}-05 & 2.86 \mathrm{E}-05 & 1.12 \mathrm{E}-05 \\ \text { CU } & 8.71 \mathrm{E}-07 & 7.27 \mathrm{E}-07 & 3.34 \mathrm{E}-07 & 5.51 \mathrm{E}-07 & 3.92 \mathrm{E}-07 & 3.54 \mathrm{E}-07 & 3.68 \mathrm{E}-07 \\ \text { FE } & 1.07 \mathrm{E}-05 & 2.34 \mathrm{E}-05 & 2.30 \mathrm{E}-05 & 3.58 \mathrm{E}-06 & 3.95 \mathrm{E}-05 & 6.73 \mathrm{E}-06 & 2.72 \mathrm{E}-04 \\ \text { HG } & 1.39 \mathrm{E}-06 & 3.10 \mathrm{E}-06 & 1.99 \mathrm{E}-06 & 1.12 \mathrm{E}-06 & 3.78 \mathrm{E}-06 & 9.28 \mathrm{E}-07 & 1.55 \mathrm{E}-06 \\ \text { MN } & 4.96 \mathrm{E}-06 & 6.10 \mathrm{E}-06 & 5.92 \mathrm{E}-06 & 3.94 \mathrm{E}-06 & 5.07 \mathrm{E}-06 & 4.00 \mathrm{E}-06 & 3.47 \mathrm{E}-05 \\ \text { NI } & 4.80 \mathrm{E}-06 & 3.61 \mathrm{E}-06 & 4.87 \mathrm{E}-06 & 1.37 \mathrm{E}-06 & 2.20 \mathrm{E}-06 & 2.26 \mathrm{E}-06 & 5.18 \mathrm{E}-07 \\ \text { NO3 } & & & & & & & \\ \text { O\&G } & & & & & & & \\ \text { PB } & 9.23 \mathrm{E}-06 & 7.18 \mathrm{E}-06 & 6.60 \mathrm{E}-06 & 3.50 \mathrm{E}-06 & 2.10 \mathrm{E}-06 & 1.59 \mathrm{E}-05 & 1.05 \mathrm{E}-05 \\ \text { PERCL } & & & & & & & \\ \text { PHE } & & & & & & & \\ \text { PHOS } & 1.63 \mathrm{E}-01 & 4.88 \mathrm{E}-02 & 1.16 \mathrm{E}-01 & 2.39 \mathrm{E}-02 & 2.69 \mathrm{E}-02 & 2.25 \mathrm{E}-02 & 7.21 \mathrm{E}-02 \\ \text { SE } & 1.30 \mathrm{E}-05 & 7.43 \mathrm{E}-06 & 9.56 \mathrm{E}-06 & 7.30 \mathrm{E}-06 & 7.20 \mathrm{E}-06 & 6.12 \mathrm{E}-06 & 1.65 \mathrm{E}-05 \\ \text { SO4 } & & & & & & & \\ \text { TCE } & & & & & & & \\ \text { TRICL } & & & & & & & \end{array}$


Table 8

Calculated Exposures from Dermal Contact with Carcinogenic Contaminants in Water While Swimming

\begin{tabular}{|c|c|c|c|c|c|c|c|}
\hline Contaminant & $\begin{array}{c}1989 \\
\mathrm{mg} / \mathrm{kg} \text {-day }\end{array}$ & $\begin{array}{c}1990 \\
\mathrm{mg} / \mathrm{kg} \text {-day }\end{array}$ & $\begin{array}{c}1991 \\
\mathrm{mg} / \mathrm{kg} \text {-day }\end{array}$ & $\begin{array}{c}1992 \\
\mathrm{mg} / \mathrm{kg} \text {-day }\end{array}$ & $\begin{array}{c}1993 \\
\mathrm{mg} / \mathrm{kg} \text {-day }\end{array}$ & $\begin{array}{c}1994 \\
\mathrm{mg} / \mathrm{kg} \text {-day }\end{array}$ & $\begin{array}{c}1995 \\
\mathrm{mg} / \mathrm{kg} \text {-day }\end{array}$ \\
\hline AG & $3.79 \mathrm{E}-11$ & $6.93 \mathrm{E}-11$ & $2.79 \mathrm{E}-11$ & $2.13 \mathrm{E}-11$ & $9.42 \mathrm{E}-09$ & $1.78 \mathrm{E}-11$ & $9.52 \mathrm{E}-09$ \\
\hline $\mathrm{AL}$ & $1.62 \mathrm{E}-09$ & $1.49 \mathrm{E}-09$ & $1.45 \mathrm{E}-09$ & $6.49 \mathrm{E}-10$ & $4.37 \mathrm{E}-09$ & $4.51 \mathrm{E}-09$ & $7.18 \mathrm{E}-09$ \\
\hline AN & $3.26 \mathrm{E}-10$ & $1.80 \mathrm{E}-10$ & $6.08 \mathrm{E}-10$ & $9.41 \mathrm{E}-10$ & 4.30E-10 & $5.63 \mathrm{E}-10$ & $1.18 \mathrm{E}-09$ \\
\hline AS & 2.27E-10 & $1.30 \mathrm{E}-10$ & $1.67 \mathrm{E}-10$ & $1.28 \mathrm{E}-10$ & $2.52 \mathrm{E}-10$ & $1.78 \mathrm{E}-10$ & 5.77E-10 \\
\hline BA & $1.19 \mathrm{E}-09$ & $5.37 \mathrm{E}-10$ & $6.97 \mathrm{E}-10$ & $4.64 \mathrm{E}-10$ & $6.39 \mathrm{E}-10$ & $4.71 \mathrm{E}-10$ & $3.86 \mathrm{E}-10$ \\
\hline $\mathrm{BEN}$ & $9.68 \mathrm{E}-14$ & $6.68 \mathrm{E}-15$ & $2.84 \mathrm{E}-14$ & $8.45 \mathrm{E}-15$ & $7.30 \mathrm{E}-15$ & $3.48 \mathrm{E}-15$ & $1.42 \mathrm{E}-14$ \\
\hline $\mathrm{C}$ & $1.39 \mathrm{E}-09$ & $1.55 \mathrm{E}-10$ & $1.92 \mathrm{E}-10$ & $1.22 \mathrm{E}-10$ & $1.70 \mathrm{E}-10$ & $1.14 \mathrm{E}-10$ & $5.09 \mathrm{E}-11$ \\
\hline CD & $7.58 \mathrm{E}-10$ & $4.33 \mathrm{E}-10$ & $5.57 \mathrm{E}-10$ & $4.26 \mathrm{E}-10$ & $5.04 \mathrm{E}-10$ & $3.57 \mathrm{E}-10$ & $1.44 \mathrm{E}-10$ \\
\hline CHL & $7.26 \mathrm{E}-11$ & $3.54 \mathrm{E}-11$ & $4.53 \mathrm{E}-11$ & $4.78 \mathrm{E}-11$ & $1.44 \mathrm{E}-09$ & 1.92E-09 & $1.44 \mathrm{E}-09$ \\
\hline $\mathrm{CN}$ & $1.22 \mathrm{E}-13$ & $2.84 \mathrm{E}-14$ & $2.13 \mathrm{E}-14$ & $4.56 \mathrm{E}-14$ & $5.40 \mathrm{E}-14$ & $3.83 \mathrm{E}-14$ & $9.60 \mathrm{E}-14$ \\
\hline CR & $2.10 \mathrm{E}-09$ & $9.53 \mathrm{E}-10$ & $1.19 \mathrm{E}-09$ & $9.18 \mathrm{E}-10$ & $1.59 \mathrm{E}-09$ & $8.36 \mathrm{E}-10$ & $3.26 \mathrm{E}-10$ \\
\hline $\mathrm{CU}$ & $1.02 \mathrm{E}-10$ & 8.49E-11 & $3.91 \mathrm{E}-11$ & $6.44 \mathrm{E}-11$ & $4.58 \mathrm{E}-11$ & $4.14 \mathrm{E}-11$ & $4.30 \mathrm{E}-11$ \\
\hline $\mathrm{FE}$ & $6.26 \mathrm{E}-10$ & $1.36 \mathrm{E}-09$ & $1.34 \mathrm{E}-09$ & $2.09 \mathrm{E}-10$ & $2.31 \mathrm{E}-09$ & $3.93 \mathrm{E}-10$ & $1.59 \mathrm{E}-08$ \\
\hline HG & $8.14 \mathrm{E}-12$ & $1.81 \mathrm{E}-11$ & $1.17 \mathrm{E}-11$ & $6.53 \mathrm{E}-12$ & $2.21 \mathrm{E}-11$ & $5.42 \mathrm{E}-12$ & $9.08 \mathrm{E}-12$ \\
\hline MN & $7.25 \mathrm{E}-11$ & 8.91E-11 & $8.65 \mathrm{E}-11$ & $5.75 \mathrm{E}-11$ & $7.40 \mathrm{E}-11$ & $5.85 \mathrm{E}-11$ & $5.06 \mathrm{E}-10$ \\
\hline NI & $2.80 \mathrm{E}-10$ & $2.11 \mathrm{E}-10$ & $2.84 \mathrm{E}-10$ & $8.00 \mathrm{E}-11$ & $1.29 \mathrm{E}-10$ & $1.32 \mathrm{E}-10$ & $3.02 \mathrm{E}-11$ \\
\hline NO3 & $2.99 \mathrm{E}-08$ & $1.17 \mathrm{E}-08$ & $1.14 \mathrm{E}-08$ & $1.52 \mathrm{E}-08$ & $1.91 \mathrm{E}-08$ & $2.14 \mathrm{E}-08$ & $1.67 \mathrm{E}-08$ \\
\hline O\&G & 5.26E-07 & $2.05 \mathrm{E}-07$ & $5.90 \mathrm{E}-07$ & 5.41E-07 & $1.36 \mathrm{E}-07$ & $2.58 \mathrm{E}-07$ & $1.30 \mathrm{E}-07$ \\
\hline PB & $5.39 \mathrm{E}-10$ & $4.19 \mathrm{E}-10$ & $3.86 \mathrm{E}-10$ & $2.04 \mathrm{E}-10$ & $1.23 \mathrm{E}-10$ & $9.27 \mathrm{E}-10$ & $6.12 \mathrm{E}-10$ \\
\hline PERCL & $5.06 \mathrm{E}-12$ & $3.35 \mathrm{E}-12$ & $3.60 \mathrm{E}-12$ & $4.30 \mathrm{E}-12$ & $5.94 \mathrm{E}-12$ & 4.17E-12 & $9.77 \mathrm{E}-12$ \\
\hline PHE & $2.73 \mathrm{E}-13$ & $3.01 \mathrm{E}-14$ & $6.74 \mathrm{E}-14$ & $3.89 \mathrm{E}-14$ & $1.46 \mathrm{E}-14$ & $2.09 \mathrm{E}-14$ & $6.46 \mathrm{E}-14$ \\
\hline PHOS & $9.51 \mathrm{E}-09$ & $2.85 \mathrm{E}-09$ & $6.77 \mathrm{E}-09$ & $1.40 \mathrm{E}-09$ & $1.57 \mathrm{E}-09$ & $1.31 \mathrm{E}-09$ & $4.21 \mathrm{E}-09$ \\
\hline SE & $4.54 \mathrm{E}-10$ & $2.60 \mathrm{E}-10$ & $3.35 \mathrm{E}-10$ & $2.55 \mathrm{E}-10$ & $2.52 \mathrm{E}-10$ & $2.14 \mathrm{E}-10$ & 5.77E-10 \\
\hline SO4 & $4.46 \mathrm{E}-07$ & $5.07 \mathrm{E}-08$ & $1.54 \mathrm{E}-08$ & 3.58E-09 & $1.47 \mathrm{E}-08$ & $4.64 \mathrm{E}-09$ & $7.47 \mathrm{E}-08$ \\
\hline TCE & $4.32 \mathrm{E}-12$ & 2.37E-12 & $2.48 \mathrm{E}-12$ & $3.02 \mathrm{E}-12$ & $2.45 \mathrm{E}-12$ & $2.23 \mathrm{E}-12$ & $7.54 \mathrm{E}-12$ \\
\hline TRICL & $1.39 \mathrm{E}-11$ & $4.91 \mathrm{E}-12$ & $3.60 \mathrm{E}-12$ & $5.40 \mathrm{E}-12$ & $7.09 \mathrm{E}-12$ & $1.80 \mathrm{E}-11$ & $9.20 \mathrm{E}-12$ \\
\hline U308 & $1.42 \mathrm{E}-10$ & $1.87 \mathrm{E}-10$ & $4.80 \mathrm{E}-11$ & $6.51 \mathrm{E}-11$ & $1.77 \mathrm{E}-10$ & $7.23 \mathrm{E}-11$ & $1.86 \mathrm{E}-09$ \\
\hline $\mathrm{ZN}$ & $3.23 \mathrm{E}-10$ & $4.64 \mathrm{E}-10$ & $3.37 \mathrm{E}-10$ & $2.82 \mathrm{E}-10$ & $2.93 \mathrm{E}-10$ & $3.31 \mathrm{E}-10$ & 4.37E-10 \\
\hline
\end{tabular}


Table 9

Calculated Exposures from Dermal Contact with Noncarcinogenic Contaminants in Water While Swimming

\begin{tabular}{|c|c|c|c|c|c|c|c|}
\hline Contaminant & $\begin{array}{c}1989 \\
\mathrm{mg} / \mathrm{kg} \text {-day }\end{array}$ & $\begin{array}{c}1990 \\
\mathrm{mg} / \mathrm{kg} \text {-day }\end{array}$ & $\begin{array}{c}1991 \\
\mathrm{mg} / \mathrm{kg}-\text { day }\end{array}$ & $\begin{array}{c}1992 \\
\mathrm{mg} / \mathrm{kg} \text {-day }\end{array}$ & $\begin{array}{c}1993 \\
\mathrm{mg} / \mathrm{kg} \text {-day }\end{array}$ & $\begin{array}{c}1994 \\
\mathrm{mg} / \mathrm{kg} \text {-day }\end{array}$ & $\begin{array}{c}1995 \\
\mathrm{mg} / \mathrm{kg} \text {-da }\end{array}$ \\
\hline $\mathrm{AG}$ & $8.84 \mathrm{E}-11$ & $1.62 \mathrm{E}-10$ & $6.50 \mathrm{E}-11$ & $4.97 \mathrm{E}-11$ & $2.20 \mathrm{E}-08$ & $4.16 \mathrm{E}-11$ & $2.22 \mathrm{E}-$ \\
\hline $\mathrm{AL}$ & 3.79E-09 & $3.48 \mathrm{E}-09$ & $3.38 \mathrm{E}-09$ & $1.51 \mathrm{E}-09$ & $1.02 \mathrm{E}-08$ & $1.05 \mathrm{E}-08$ & $1.67 \mathrm{E}-0$ \\
\hline AN & $7.60 \mathrm{E}-10$ & $4.20 \mathrm{E}-10$ & $1.42 \mathrm{E}-09$ & $2.20 \mathrm{E}-09$ & $1.00 \mathrm{E}-09$ & $1.31 \mathrm{E}-09$ & $2.76 \mathrm{E}-09$ \\
\hline AS & $5.30 \mathrm{E}-10$ & $3.03 \mathrm{E}-10$ & $3.90 \mathrm{E}-10$ & $2.98 \mathrm{E}-10$ & $5.88 \mathrm{E}-10$ & $4.16 \mathrm{E}-10$ & $1.35 \mathrm{E}-0$ \\
\hline BA & 2.77E-09 & $1.25 \mathrm{E}-09$ & $1.63 \mathrm{E}-09$ & $1.08 \mathrm{E}-09$ & $1.49 \mathrm{E}-09$ & $1.10 \mathrm{E}-09$ & $9.02 \mathrm{E}-10$ \\
\hline BEN & $2.26 \mathrm{E}-13$ & $1.56 \mathrm{E}-14$ & $6.63 \mathrm{E}-14$ & $1.97 \mathrm{E}-14$ & $1.70 \mathrm{E}-14$ & $8.13 \mathrm{E}-15$ & $3.31 \mathrm{E}-14$ \\
\hline $\mathrm{C}$ & $3.25 \mathrm{E}-09$ & $3.61 \mathrm{E}-10$ & $4.48 \mathrm{E}-10$ & $2.84 \mathrm{E}-10$ & $3.96 \mathrm{E}-10$ & $2.66 \mathrm{E}-10$ & $1.19 \mathrm{E}-1$ \\
\hline CD & 1.77E-09 & $1.01 \mathrm{E}-09$ & $1.30 \mathrm{E}-09$ & $9.93 \mathrm{E}-10$ & $1.18 \mathrm{E}-09$ & 8.34E-10 & $3.37 \mathrm{E}-10$ \\
\hline CHL & $1.69 \mathrm{E}-10$ & $8.26 \mathrm{E}-11$ & $1.06 \mathrm{E}-10$ & $1.12 \mathrm{E}-10$ & 3.37E-09 & $4.48 \mathrm{E}-09$ & $3.36 \mathrm{E}-09$ \\
\hline $\mathrm{CN}$ & $2.84 \mathrm{E}-13$ & $6.63 \mathrm{E}-14$ & $4.97 \mathrm{E}-14$ & $1.06 \mathrm{E}-13$ & 1.261 & 8.94E-14 & $2.24 \mathrm{E}-13$ \\
\hline CR & 4.91E-09 & 2.22E-09 & 2.78E-09 & 2.14E-09 & $3.71 \mathrm{E}-09$ & $1.95 \mathrm{E}-09$ & $7.61 \mathrm{E}-1$ \\
\hline CU & $2.38 \mathrm{E}-10$ & $1.98 \mathrm{E}-10$ & $9.12 \mathrm{E}-11$ & $1.50 \mathrm{E}-10$ & $1.07 \mathrm{E}-10$ & $9.66 \mathrm{E}-11$ & $1.00 \mathrm{E}-10$ \\
\hline FE & $1.46 \mathrm{E}-09$ & $3.18 \mathrm{E}-09$ & $3.14 \mathrm{E}-09$ & $4.88 \mathrm{E}-10$ & $5.39 \mathrm{E}-09$ & $9.17 \mathrm{E}-10$ & $3.71 \mathrm{E}-0$ \\
\hline HG & $1.90 \mathrm{E}-11$ & $4.22 \mathrm{E}-11$ & $2.72 \mathrm{E}-11$ & $1.52 \mathrm{E}-11$ & $5.15 \mathrm{E}-11$ & $1.27 \mathrm{E}-11$ & $2.12 \mathrm{E}-1$ \\
\hline MN & $1.69 \mathrm{E}-10$ & $2.08 \mathrm{E}-10$ & $2.02 \mathrm{E}-10$ & $1.34 \mathrm{E}-10$ & $1.73 \mathrm{E}-10$ & $1.36 \mathrm{E}-10$ & $1.18 \mathrm{E}-0$ \\
\hline NI & $6.54 \mathrm{E}-10$ & $4.93 \mathrm{E}-10$ & 6.64 & 1.87 & 3.00 & $3.09 \mathrm{E}-10$ & $7.06 \mathrm{E}-1$ \\
\hline NO3 & $6.97 \mathrm{E}-08$ & 2.73E-08 & $2.66 \mathrm{E}-08$ & $3.55 \mathrm{E}-08$ & 4.4 & 4.9 & $3.89 \mathrm{E}-\mathrm{C}$ \\
\hline O\&G & $1.23 \mathrm{E}-06$ & $4.79 \mathrm{E}-07$ & $1.38 \mathrm{E}-06$ & $1.26 \mathrm{E}-06$ & $3.18 \mathrm{E}-07$ & $6.02 \mathrm{E}-07$ & $3.03 \mathrm{E}-\mathrm{C}$ \\
\hline PB & 1.26 & $9.78 \mathrm{E}-10$ & $9.00 \mathrm{E}-10$ & $4.77 \mathrm{E}-10$ & $2.87 \mathrm{E}-10$ & 2.16E-09 & $1.43 \mathrm{E}-\mathrm{C}$ \\
\hline PERCL & 1.18 & $7.82 \mathrm{E}-12$ & 8.41 & $1.00 \mathrm{E}-11$ & $1.39 \mathrm{E}-11$ & $9.72 \mathrm{E}-12$ & $2.28 \mathrm{E}-1$ \\
\hline PHE & 6.37E-13 & $7.02 \mathrm{E}-14$ & $1.57 \mathrm{E}-13$ & $9.07 \mathrm{E}-14$ & $3.41 \mathrm{E}-14$ & $4.88 \mathrm{E}-14$ & $1.51 \mathrm{E}-$ \\
\hline PHOS & $2.22 \mathrm{E}-08$ & $6.65 \mathrm{E}-09$ & $1.58 \mathrm{E}-08$ & $3.26 \mathrm{E}-09$ & $3.67 \mathrm{E}-09$ & $3.06 \mathrm{E}-09$ & $9.83 \mathrm{E}-\mathrm{C}$ \\
\hline SE & $1.06 \mathrm{E}-09$ & $6.06 \mathrm{E}-10$ & $7.81 \mathrm{E}-10$ & $5.96 \mathrm{E}-10$ & $5.88 \mathrm{E}-10$ & $4.99 \mathrm{E}-10$ & $1.35 \mathrm{E}-\mathrm{C}$ \\
\hline SO4 & $1.04 \mathrm{E}-06$ & $1.18 \mathrm{E}-07$ & $3.59 \mathrm{E}-08$ & 8.36E-09 & $3.42 \mathrm{E}-08$ & $1.08 \mathrm{E}-08$ & $1.74 \mathrm{E}-\mathrm{C}$ \\
\hline TCE & $1.01 \mathrm{E}-11$ & $5.52 \mathrm{E}-12$ & $5.80 \mathrm{E}-12$ & $7.05 \mathrm{E}-12$ & $5.71 \mathrm{E}-12$ & $5.20 \mathrm{E}-12$ & $1.76 \mathrm{E}-$ \\
\hline TRICL & $3.24 \mathrm{E}-11$ & 1.15E-11 & $8.41 \mathrm{E}-12$ & $1.26 \mathrm{E}-11$ & $1.65 \mathrm{E}-11$ & $4.19 \mathrm{E}-11$ & $2.15 \mathrm{E}-1$ \\
\hline U308 & 3.32E-10 & $4.37 \mathrm{E}-10$ & $1.12 \mathrm{E}-10$ & $1.52 \mathrm{E}-10$ & $4.14 \mathrm{E}-10$ & $1.69 \mathrm{E}-10$ & $4.34 \mathrm{E}-\mathrm{C}$ \\
\hline $\mathrm{ZN}$ & $7.54 \mathrm{E}-10$ & $1.08 \mathrm{E}-09$ & $7.87 \mathrm{E}-10$ & $6.58 \mathrm{E}-10$ & $6.83 \mathrm{E}-10$ & $7.73 \mathrm{E}-10$ & $1.02 \mathrm{E}-\mathrm{C}$ \\
\hline
\end{tabular}


Table 10

Contaminants' Toxicity

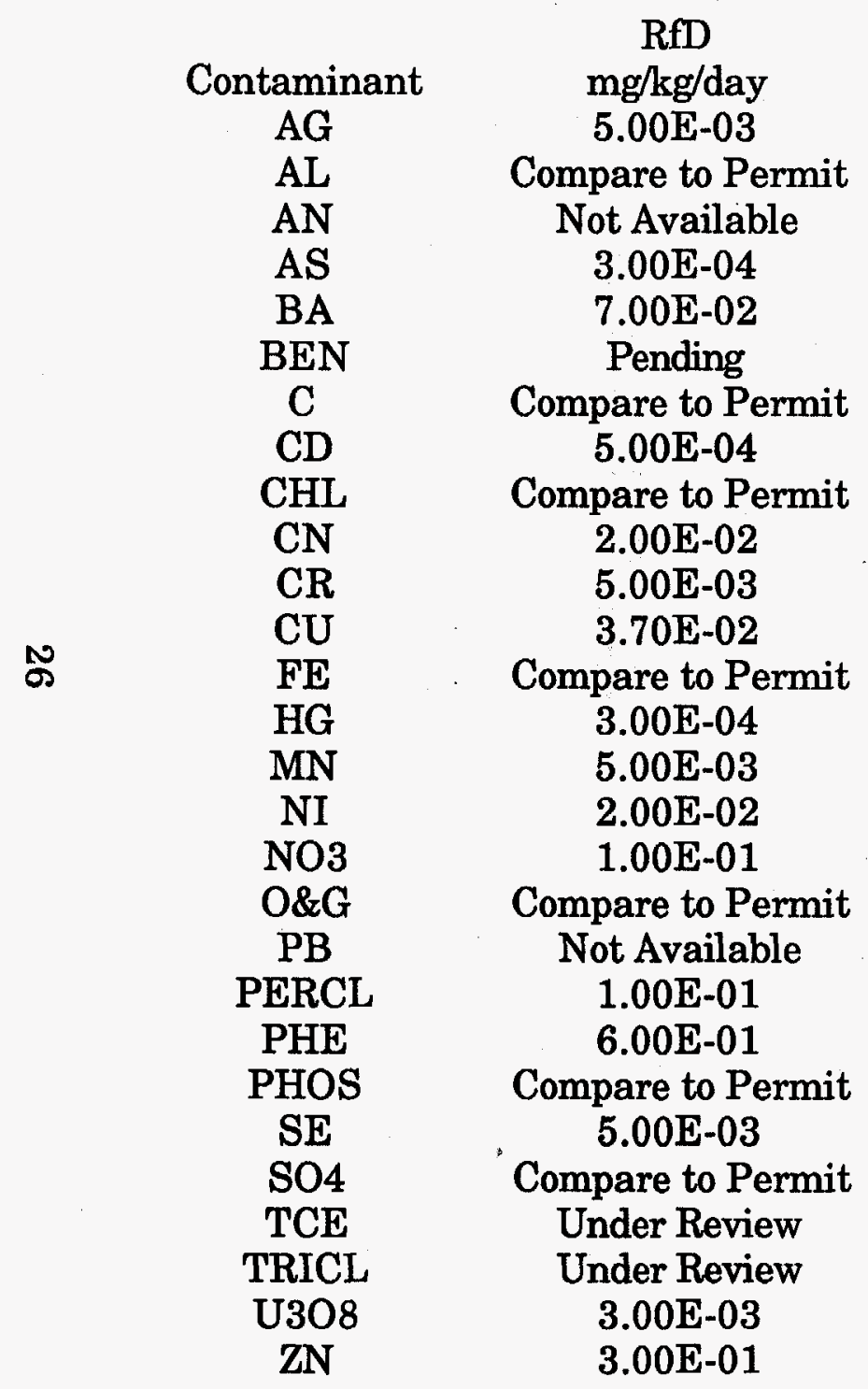

\author{
SF \\ $\mathrm{mg} / \mathrm{kg}$-day \\ Not Available \\ Compare to Permit \\ Not Available \\ $2.00 \mathrm{E}+00$ \\ Not Available \\ 3.00E-02 \\ Compare to Permit \\ Not Available \\ Compare to Permit \\ Not Available \\ Not Available \\ Not Available \\ Compare to Permit \\ None \\ Not Available \\ Not Available \\ Pending \\ Compare to Permit \\ Not Available \\ 5.00E-02 \\ None \\ Compare to Permit \\ None \\ Compare to Permit \\ Not Available \\ Under Review \\ Not Available \\ None
}


Table 11

Estimated Cancer Risks for Contaminants Released from SRS Outfalls

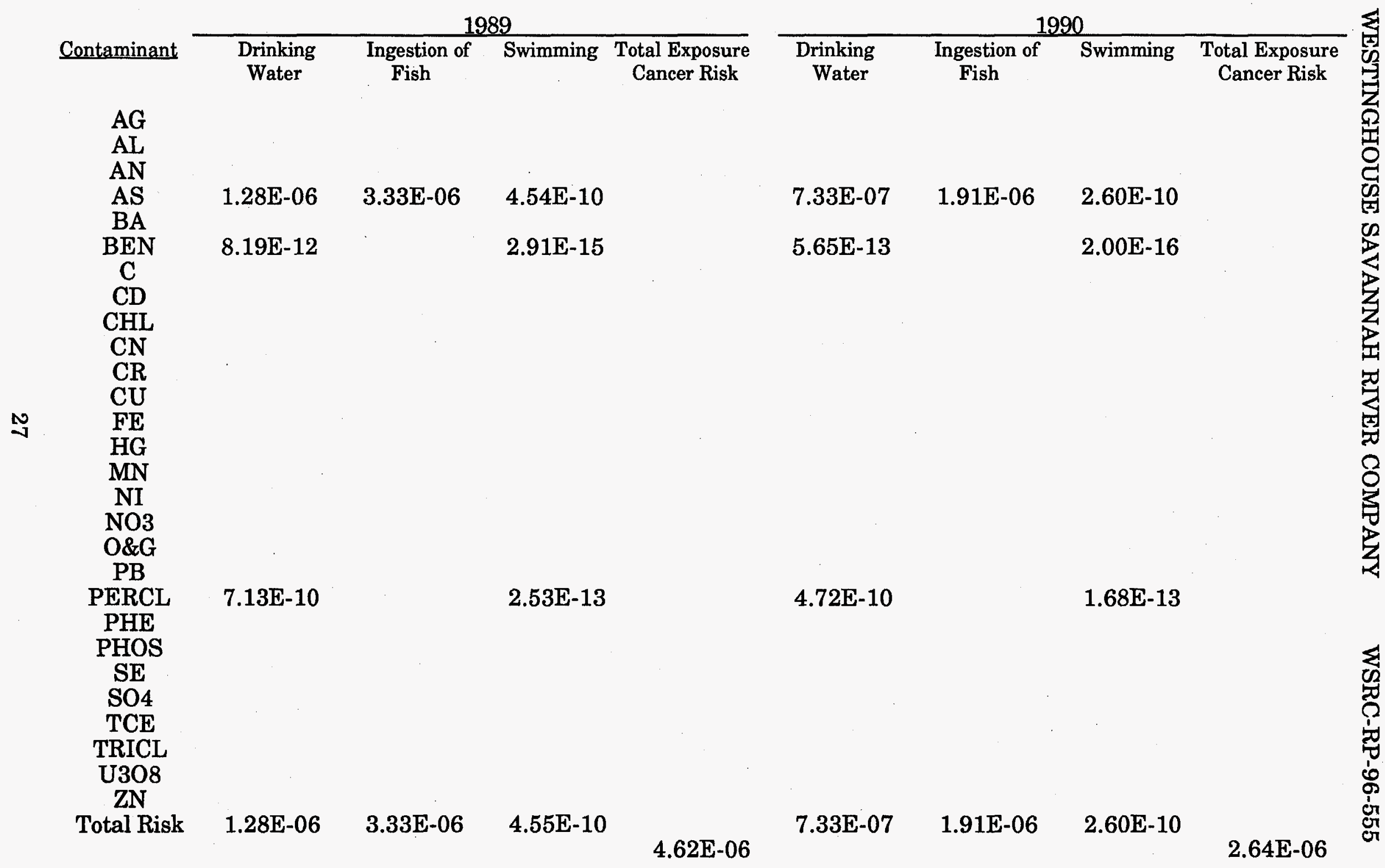


Table 11 (continued)

Estimated Cancer Risks for Contaminants Released from SRS Outfalls

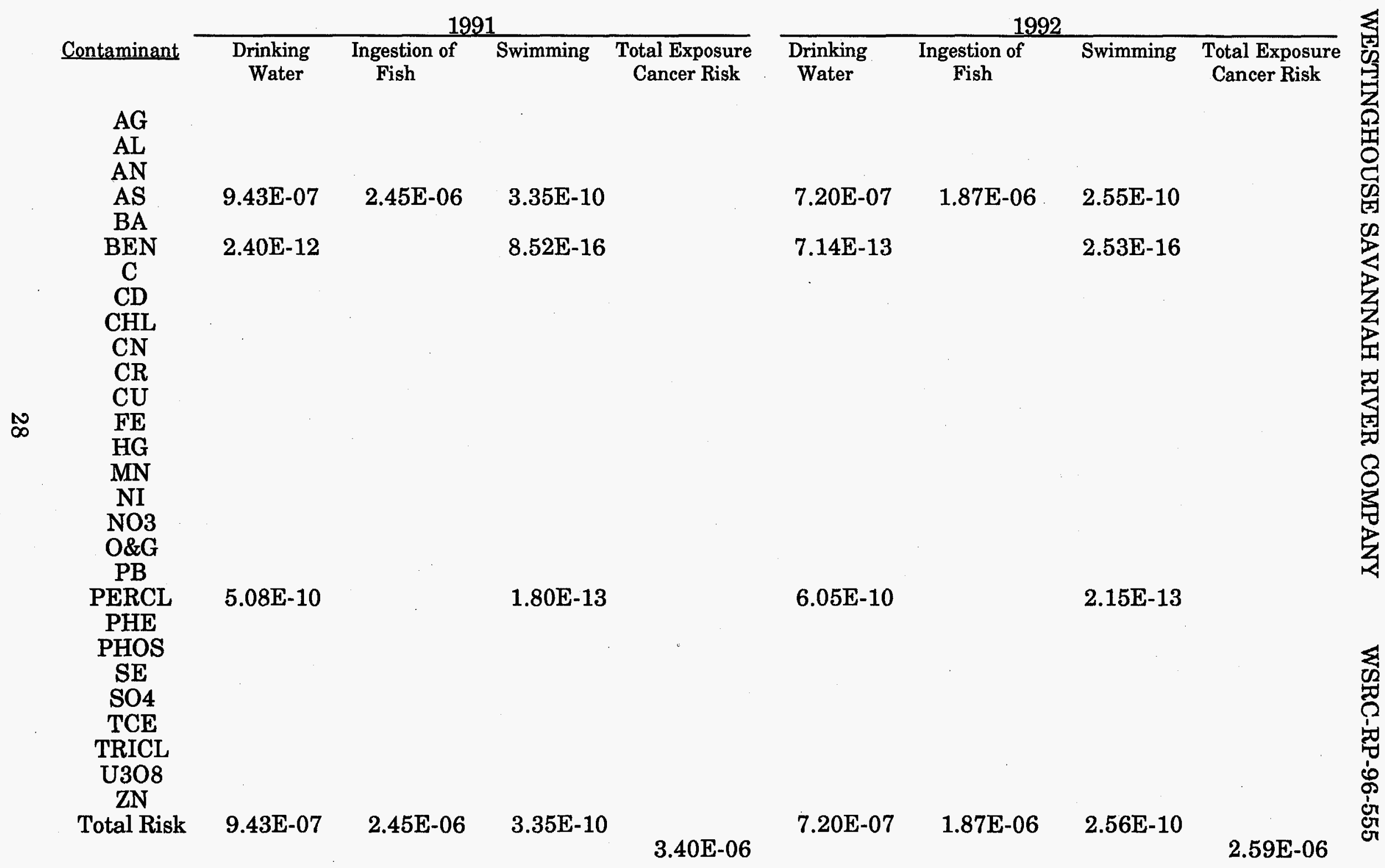


Table 11 (continued)

Estimated Cancer Risks for Contaminants Released from SRS Outfalls

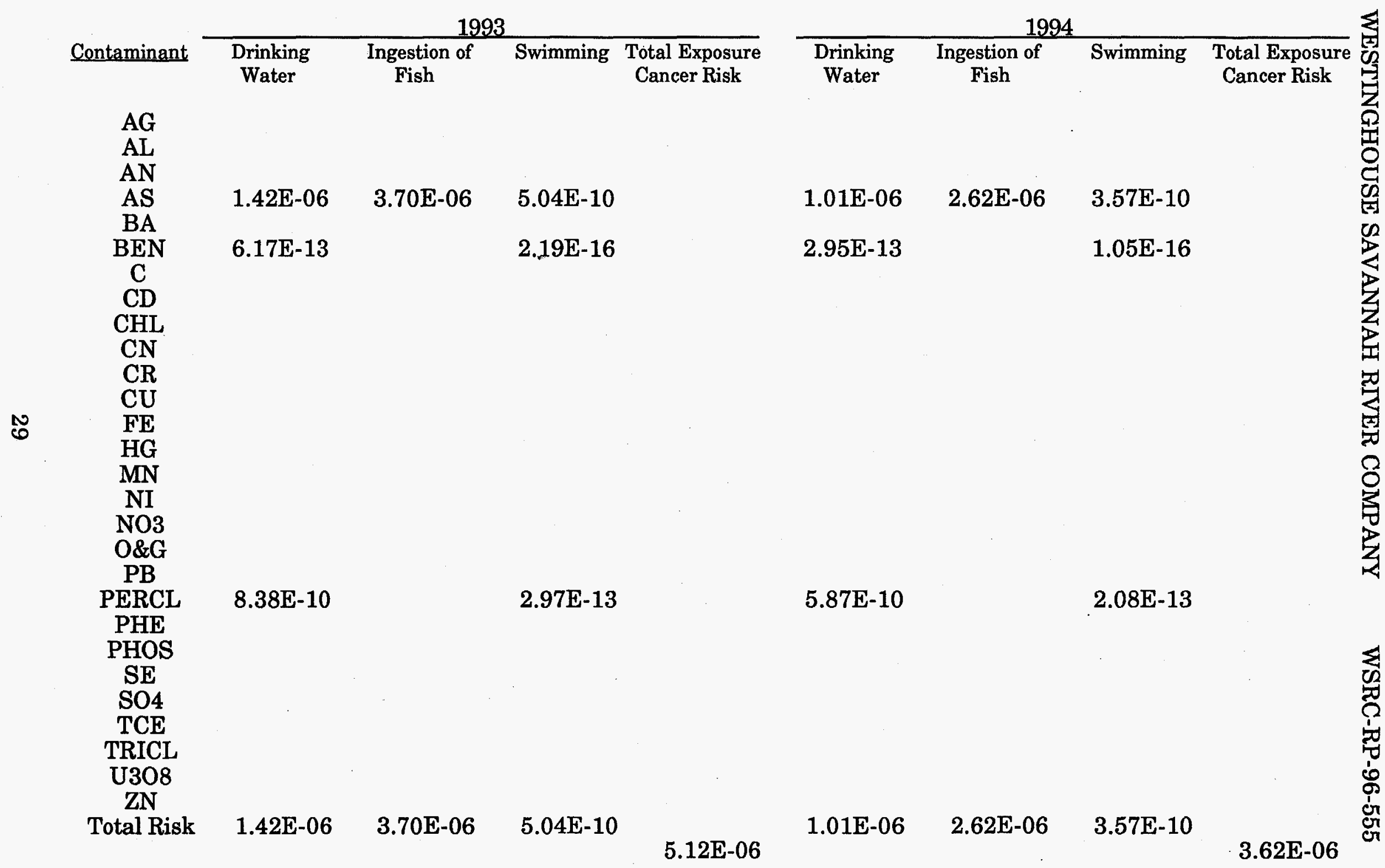


Table 11 (continued)

Estimated Cancer Risks for Contaminants Released from SRS Outfalls

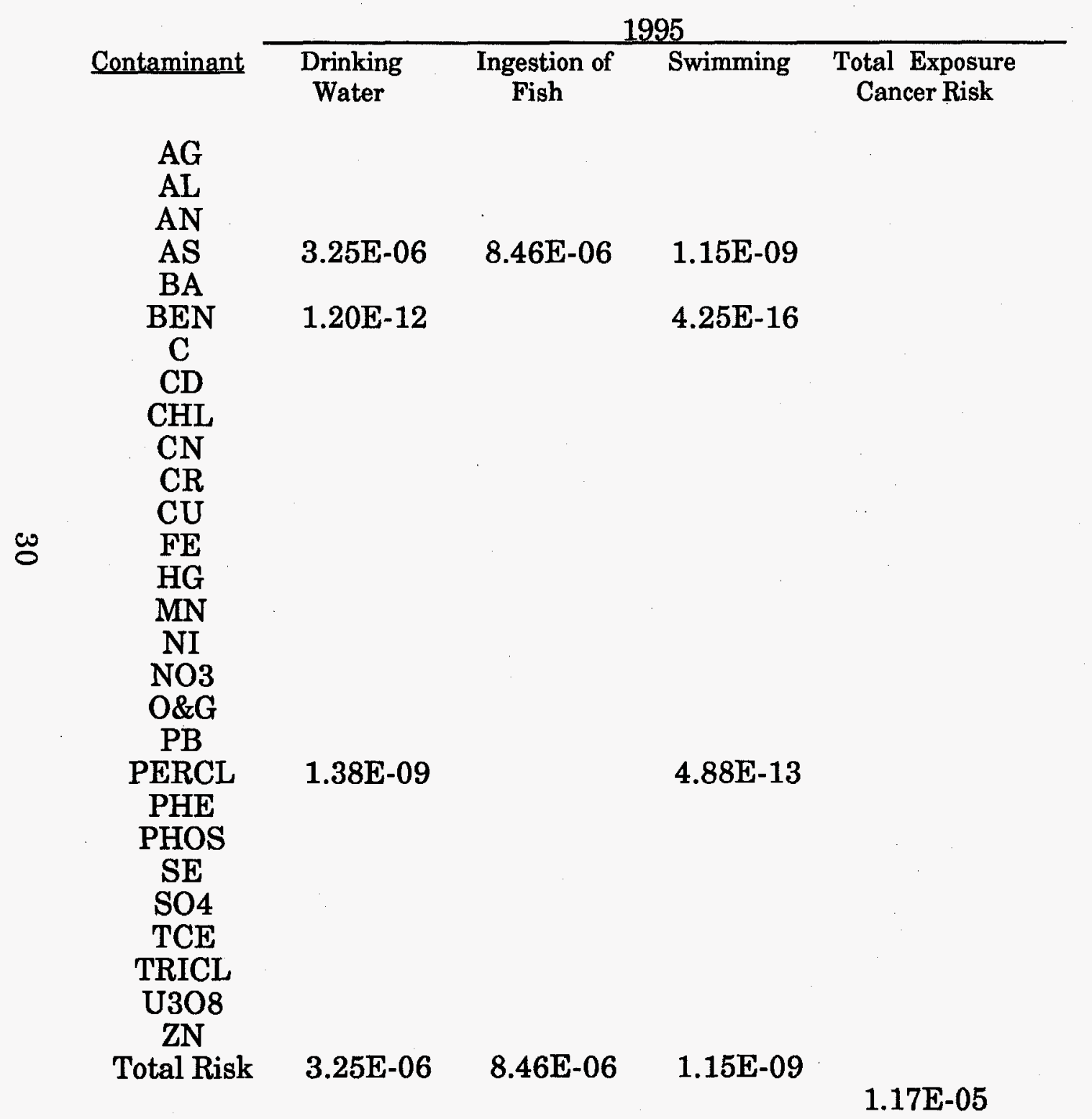


Table 12

Estimated Chronic Noncarcinogenic Hazard Quotients for Contaminants Released from SRS Outfalls

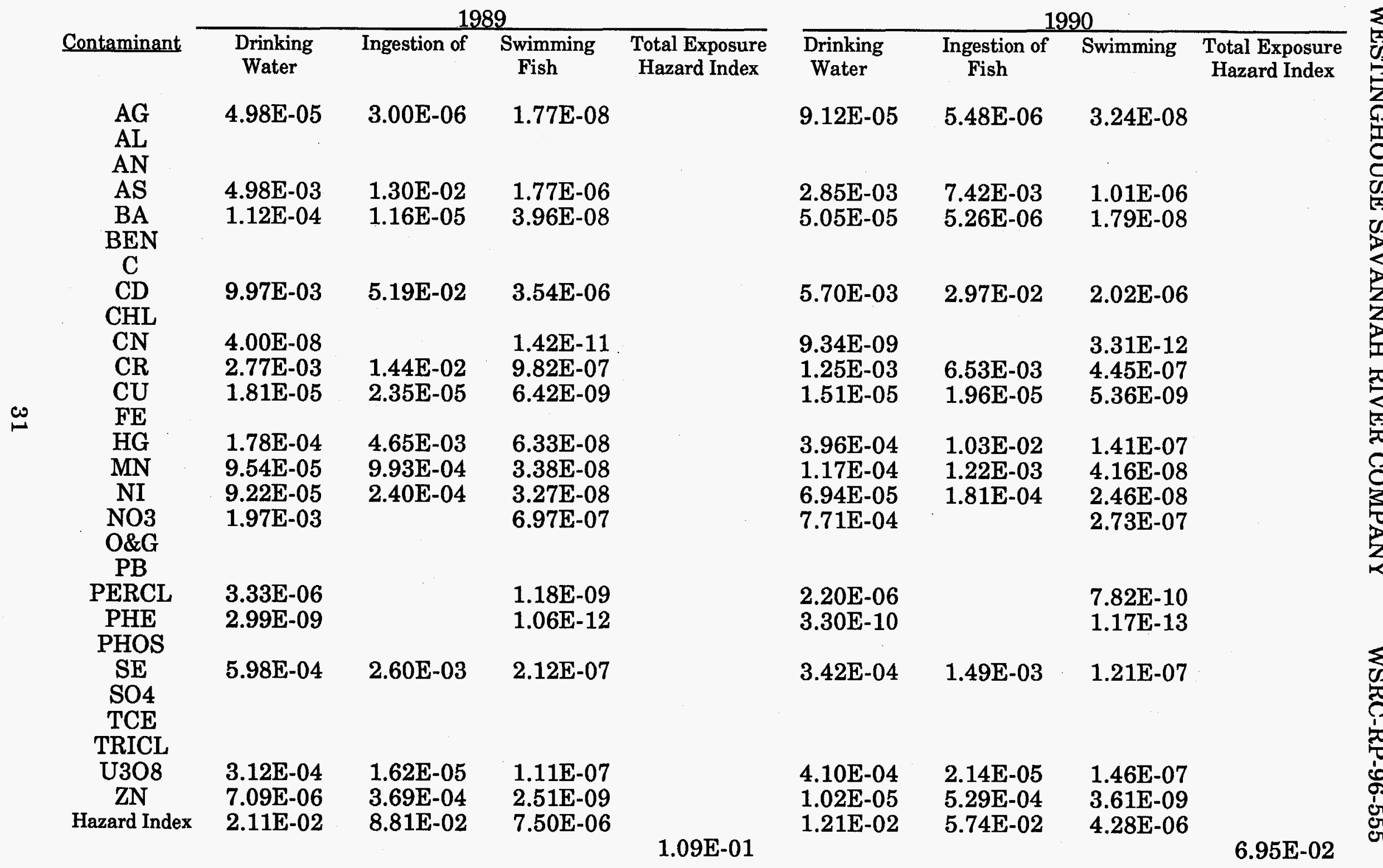


Table 12 (continued)

Estimated Chronic Noncarcinogenic Hazard Quotients for Contaminants Released from SRS Outfalls

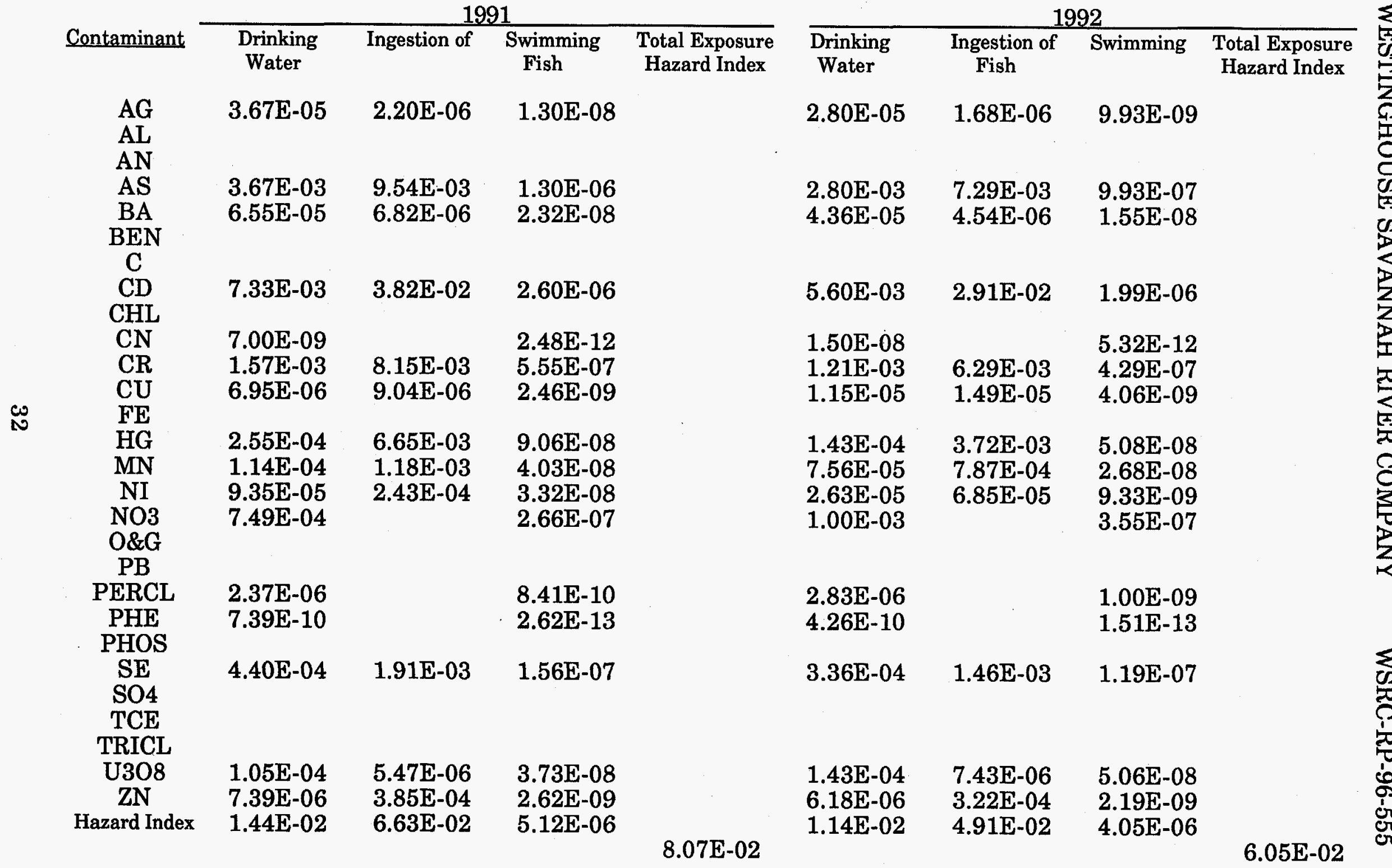


Table 12 (continued)

Estimated Chronic Noncarcinogenic Hazard Quotients for Contaminants Released from SRS Outfalls

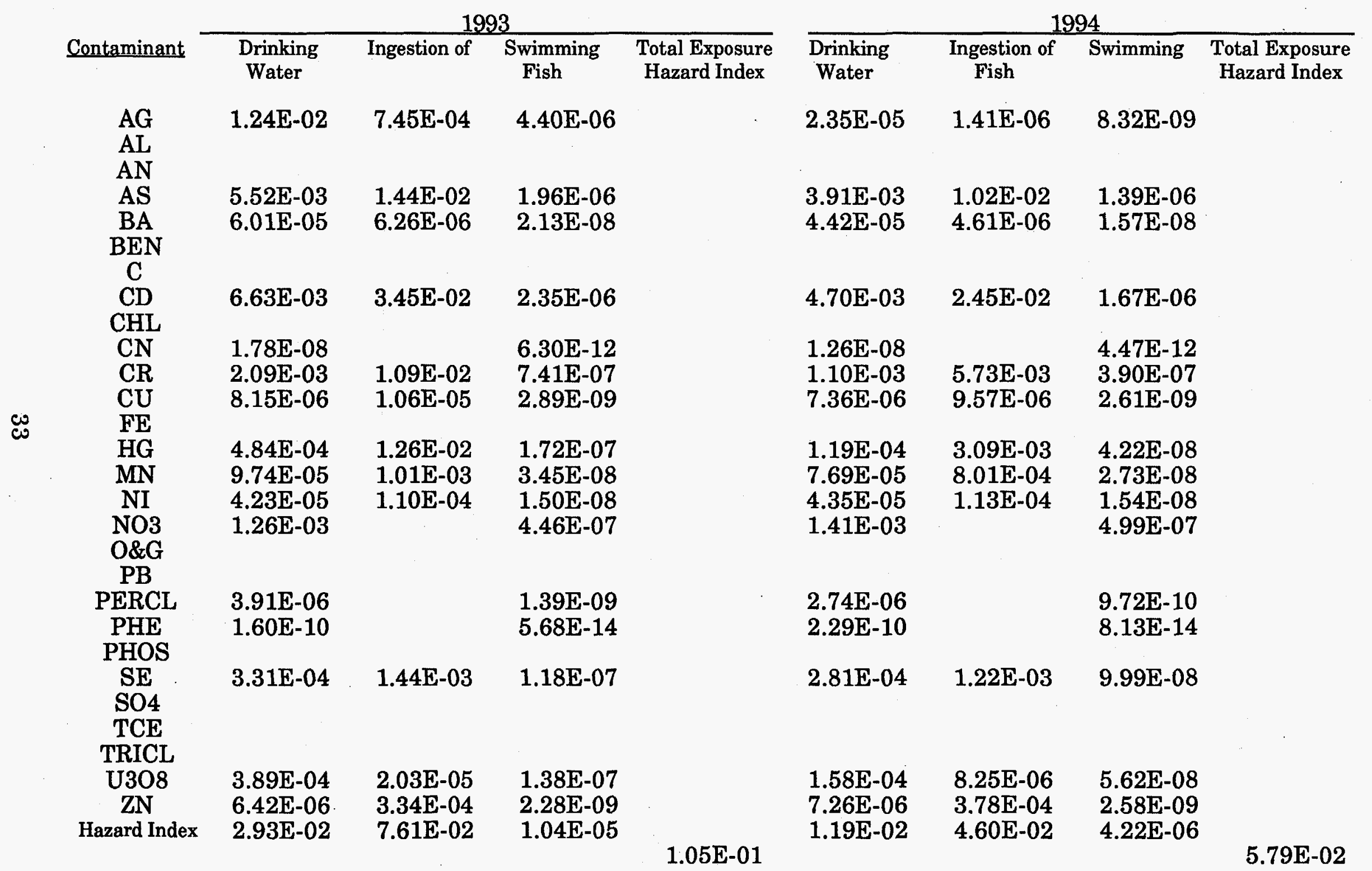


Table 12 (continued)

Estimated Chronic Noncarcinogenic Hazard Quotients for Contaminants Released from SRS Outfalls

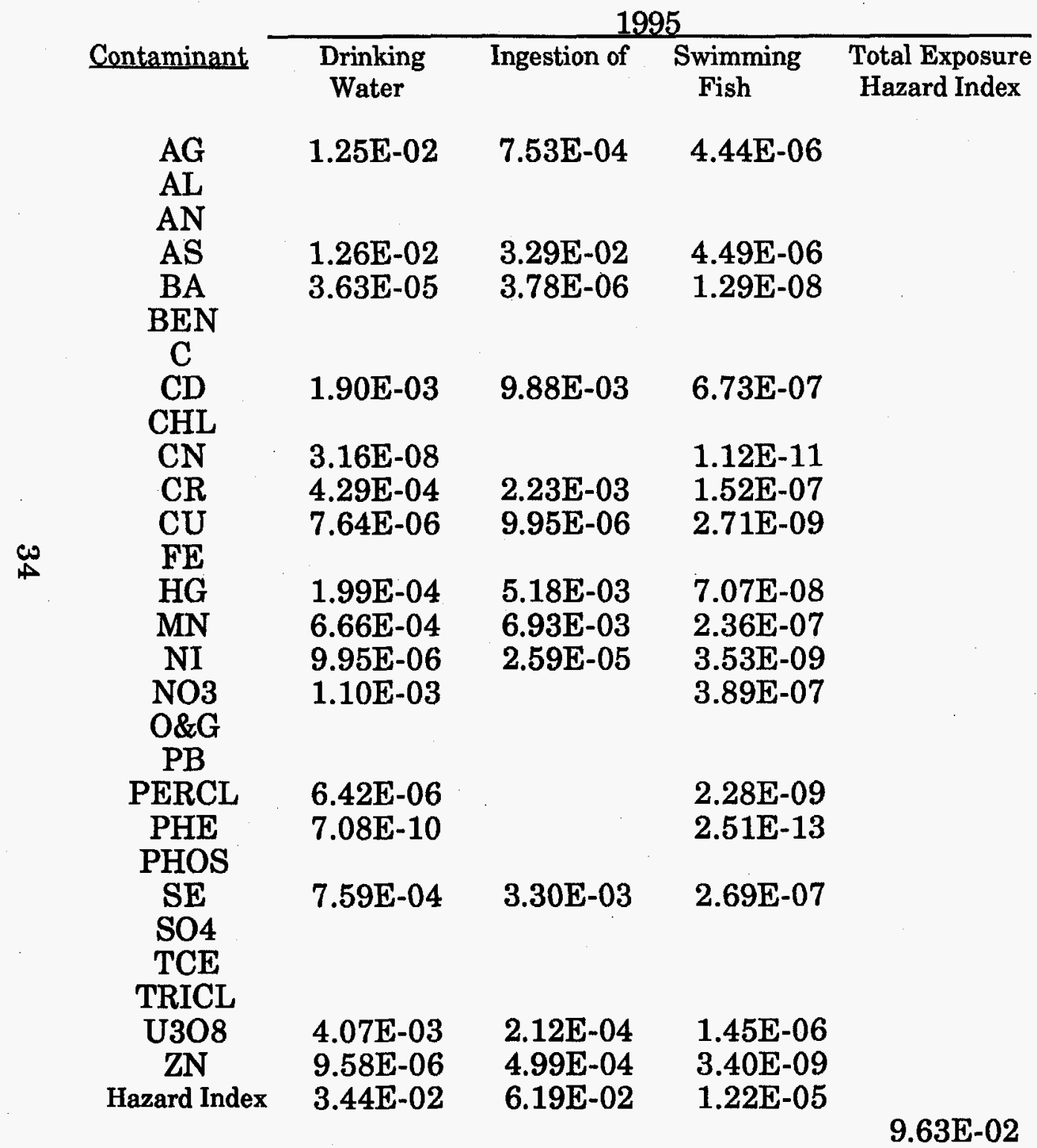


Table 13

Critical Contaminant Ranking Based on Total Exposure Cancer Risk

\begin{tabular}{|c|c|c|c|c|c|}
\hline & 989 & & 90 & & 991 \\
\hline & $\overline{\text { RISK }}$ & & RISK & & RISK \\
\hline & $4.61 \mathrm{E}-06$ & AS & 2.64E-06 & AS & $3.40 \mathrm{E}-06$ \\
\hline PERCL & $7.13 \mathrm{E}-10$ & PERCL & 4.72E-10 & PERCL & $5.08 \mathrm{E}-10$ \\
\hline BEN & $8.19 \mathrm{E}-12$ & BEN & $5.65 \mathrm{E}-13$ & BEN & $2.40 \mathrm{E}-12$ \\
\hline $\mathbf{A G}$ & & AG & & & \\
\hline & & & & $A L$ & \\
\hline AN & & AN & & AN & \\
\hline BA & & BA & & BA & \\
\hline C & & C & & C & \\
\hline CD & & CD & & CD & \\
\hline CHL & & CHL & & CHL & \\
\hline CN & & $\mathrm{CN}$ & & CN & \\
\hline CR & & $\mathrm{CR}$ & & CR & \\
\hline CU & & $\mathrm{CU}$ & & $\mathrm{CU}$ & \\
\hline FE & & $\mathrm{FE}$ & & $\mathrm{FE}$ & \\
\hline HG & & HG & & HG & \\
\hline MN & & MN & & MN & \\
\hline NI & & NI & & NI & \\
\hline NO3 & & NO3 & & NO3 & \\
\hline O\&G & & O\&G & & O\&G & \\
\hline PB & & PB & & PB & \\
\hline PHE & & PHE & & PHE & \\
\hline PHOS & & PHOS & & PHOS & \\
\hline SE & & SE & & SE & \\
\hline SO4 & & SO4 & & SO4 & \\
\hline TCE & & TCE & & TCE & \\
\hline TRICL & & TRICL & & TRICL & \\
\hline U308 & & U308 & & U308 & \\
\hline $\mathrm{ZN}$ & & $\mathrm{ZN}$ & & $\mathrm{ZN}$ & \\
\hline
\end{tabular}

\begin{tabular}{|c|c|c|c|c|}
\hline \multicolumn{2}{|c|}{1992} & \multicolumn{2}{|c|}{1993} & \\
\hline & RISK & & RISK & \\
\hline AS & $2.59 \mathrm{E}-06$ & AS & $5.12 \mathrm{E}-06$ & AS \\
\hline PERCL & $6.06 \mathrm{E}-10$ & PERCL & $8.38 \mathrm{E}-10$ & PERCL \\
\hline BEN & $7.15 \mathrm{E}-13$ & BEN & $6.17 \mathrm{E}-13$ & BEN \\
\hline $\mathbf{A G}$ & & $\mathbf{A G}$ & & AG \\
\hline AL & & AL & & AL \\
\hline AN & & AN & & AN \\
\hline BA & & BA & & BA \\
\hline C & & $\mathrm{C}$ & & $\mathrm{C}$ \\
\hline CD & & CD & & CD \\
\hline CHL & & CHL & & CHL \\
\hline CN & & $\mathrm{CN}$ & & CN \\
\hline CR & & CR & & CR \\
\hline CU & & $\mathrm{CU}$ & & $\mathbf{C U}$ \\
\hline EE & & $\mathrm{FE}$ & & $\mathbf{F E}$ \\
\hline HG & & HG & & HG \\
\hline MN & & MN & & $\mathrm{MN}$ \\
\hline NI & & NI & & NI \\
\hline NO3 & & NO3 & & NO3 \\
\hline O\&G & & O\&G & & $O \& G$ \\
\hline PB & & $\mathrm{PB}$ & & PB \\
\hline PHE & & PHE & & PHE \\
\hline PHOS & & PHOS & & \\
\hline $\mathrm{SE}$ & & $S E$ & & $\mathrm{SE}$ \\
\hline SO4 & & $\mathrm{SO} 4$ & & $\mathrm{SO} 4$ \\
\hline TCE & & TCE & & TCE \\
\hline TRICL & & TRICL & & TRICL \\
\hline U308 & & U308 & & U308 \\
\hline & & & & \\
\hline
\end{tabular}

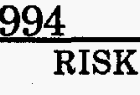

3.62E-06

PERCL 5.87E-10

2.95E-13

AI

N

CHL

CN

CU

FE

MN

NO3

$\mathrm{OEC}$

PHOS

$\mathrm{SO} 4$

U3O8

ZN

$\begin{array}{ll}\text { AS } & 1.17 \mathrm{E}-05 \\ \text { PERCL } & 1.38 \mathrm{E}-09\end{array}$

BEN 1.20E-12

$A G$

AL

BA

C

CD

CHL

CR

CU

FE

HG

MN

NO3

$O \& G$

PB

PHE

PHOS

SE

$\mathrm{SO} 4$

TCE

TRICL

U308

ZN 
Table 14

Critical Contaminant Ranking Based on Total Exposure Hazard Quotient

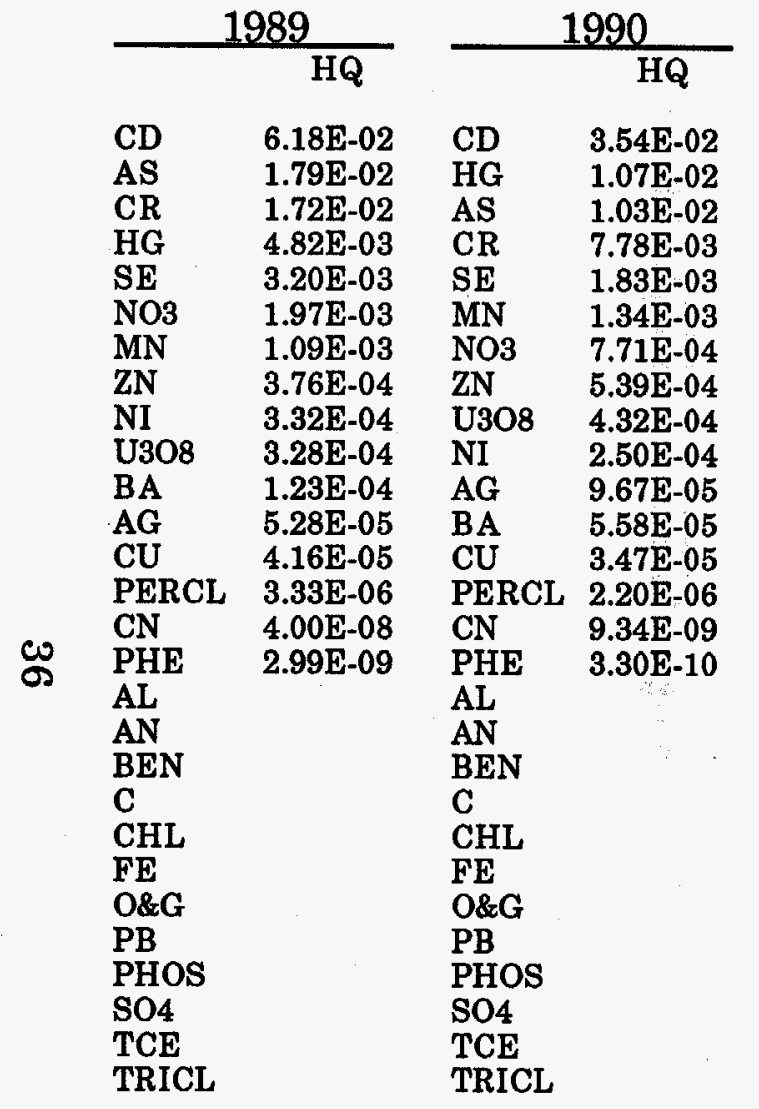

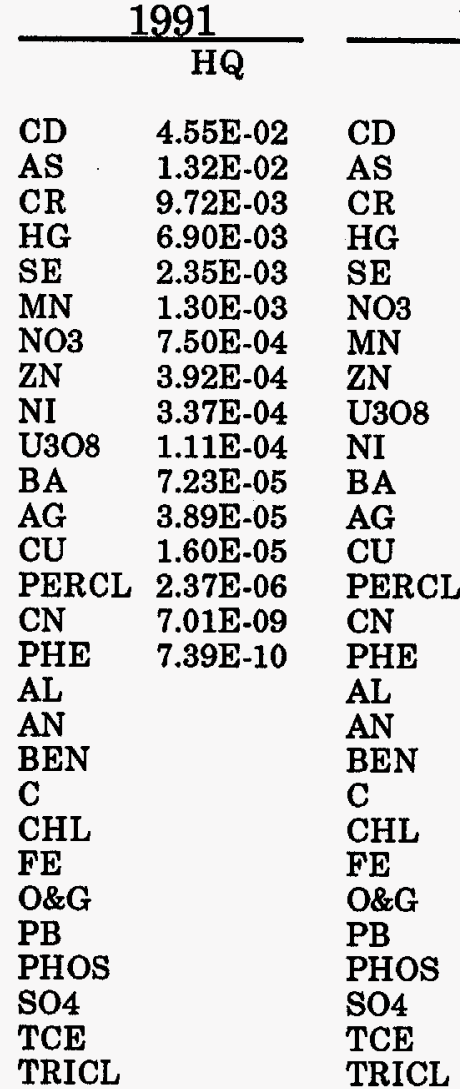

1992

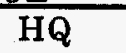

$3.47 \mathrm{E}-02$

$1.01 \mathrm{E}-02$

$7.50 \mathrm{E}-03$

$3.87 \mathrm{E}-03$

$1.80 \mathrm{E}-03$

$1.00 \mathrm{E}-03$

8.63E-04

$3.28 \mathrm{E}-04$

$1.50 \mathrm{E}-04$

$9.48 \mathrm{E}-05$

$4.81 \mathrm{E}-05$

2.97E-05

$2.64 \mathrm{E}-05$

$2.83 \mathrm{E}-06$

$1.50 \mathrm{E}-08$

$4.26 \mathrm{E}-10$

AL

BEN

CHL

O\&G

$\mathrm{PB}$

$\mathrm{SO} 4$

TRICL

\begin{tabular}{ll}
\multicolumn{2}{c}{1993} \\
\hline & HQ \\
CD & $4.12 \mathrm{E}-02$ \\
AS & $1.99 \mathrm{E}-02$ \\
AG & $1.31 \mathrm{E}-02$ \\
HG & $1.31 \mathrm{E}-02$ \\
CR & $1.30 \mathrm{E}-02$ \\
SE & $1.77 \mathrm{E}-03$ \\
NO3 & $1.26 \mathrm{E}-03$ \\
MN & $1.11 \mathrm{E}-03$ \\
U3O8 & $4.09 \mathrm{E}-04$ \\
ZN & $3.41 \mathrm{E}-04$ \\
NI & $1.52 \mathrm{E}-04$ \\
BA & $6.64 \mathrm{E}-05$ \\
CU & $1.88 \mathrm{E}-05$ \\
PERCL & $3.91 \mathrm{E}-06$ \\
CN & $1.78 \mathrm{E}-08$ \\
PHE & $1.60 \mathrm{E}-10$ \\
AL & \\
AN & \\
BEN & \\
C & \\
CHL & \\
FE & \\
O\&G & \\
PB & \\
PHOS & \\
SO4 & \\
TCE & \\
TRICL & TRICL
\end{tabular}

\begin{tabular}{l} 
CD \\
\hline AS \\
CR \\
HG \\
SE \\
NO3 \\
MN \\
ZN \\
U3O8 \\
NI \\
BA \\
AG \\
CU \\
PERCL \\
CN \\
PHE \\
AL \\
AN \\
BEN \\
C \\
CHL \\
FE \\
O\&G \\
PB \\
PHOS \\
SO4 \\
TCE \\
TRICL
\end{tabular}

HQ: Harzard Quotient 


\section{CRITICAL CONTAMINANT/CRITICAL PATHWAY ANALYSIS - SURFACE WATER TRANSPORT FOR NONRADIOACTIVE CONTAMINANTS(U)}

\section{DISTRIBUTION}
A. L. Boni, 773A
R. P. Addis, 773A
G. T. Jannik, 773A
W. H. Carlton, 773A
A. A. Simpkins, 773A
D. B. Moore-Shedrow, 773-A
J. B. Gladden, 773-42A
C. E. Murphy, Jr. 773-42A
W. A. Emel, 735-A
B. L. O'Steen, 773-A
A. J. Garrett, 773-A
D. W. Hayes, 735-A
D. P. Griggs, 773-A
C. H. Hunter, 773-A
Kuo-Fu Chen, 773-A
SRTC Records(4), 773-52A
ETG Records(5), 773-A 